\title{
Localization of high frequency waves propagating in a locally periodic medium
}

\author{
G. Allaire ${ }^{*} \quad$ L. Friz ${ }^{\dagger}$
}

February 5, 2020

\begin{abstract}
We study the homogenization and localization of high frequency waves in a locally periodic media with period $\varepsilon$. We consider initial data that are localized Bloch wave packets, i.e., that are the product of a fast oscillating Bloch wave at a given frequency $\xi$ and of a smooth envelope function whose support is concentrated at a point $x$ with length scale $\sqrt{\varepsilon}$. We assume that $(\xi, x)$ is a stationary point in the phase space of the Hamiltonian $\lambda(\xi, x)$, i.e., of the corresponding Bloch eigenvalue. Upon rescaling at size $\sqrt{\varepsilon}$ we prove that the solution of the wave equation is approximately the sum of two terms with opposite phases which are the product of the oscillating Bloch wave and of two limit envelope functions which are the solution of two Schrödinger type equations with quadratic potential. Furthermore, if the full Hessian of the Hamiltonian $\lambda(\xi, x)$ is positive definite, then localization takes place in the sense that the spectrum of each homogenized Schrödinger equation is made of a countable sequence of finite multiplicity eigenvalues with exponentially decaying eigenfunctions.
\end{abstract}

Key words: Homogenization, Bloch waves, localization.

2000 Mathematics Subject Classification: 35B27, 35J10.

\section{Introduction}

We consider the wave equation in a locally periodic medium with small period $\varepsilon>0$ and study its homogenization, i.e., its limit when $\varepsilon$ goes to zero. Our wave equation is

$$
\left\{\begin{array}{rlrl}
\rho^{\varepsilon} \frac{\partial^{2} u_{\varepsilon}}{\partial t^{2}}-\operatorname{div}\left(A^{\varepsilon} \nabla u_{\varepsilon}\right) & =0 & & \text { in } \mathbb{R}^{+} \times \mathbb{R}^{N} \\
u_{\varepsilon}(0) & =u_{\varepsilon}^{0} \quad \text { in } \mathbb{R}^{N} \\
\frac{\partial u_{\varepsilon}}{\partial t}(0) & =u_{\varepsilon}^{1} \quad \text { in } \mathbb{R}^{N}
\end{array}\right.
$$

\footnotetext{
${ }^{*}$ Centre de Mathématiques Appliquées, École Polytechnique, 91128 Palaiseau, France gregoire.allaire@polytechnique.fr

†Departamento de Ciencias Básicas, Facultad de Ciencias, Universidad del Bío-Bío. Avenida Andrés Bello s/n, Casilla 447, Chillán, Chile - lfriz@roble.fdo-may.ubiobio.cl
} 
where

$$
\rho^{\varepsilon}(x):=\rho\left(x, \frac{x}{\varepsilon}\right) \quad \text { and } \quad A^{\varepsilon}(x):=A\left(x, \frac{x}{\varepsilon}\right)
$$

and the unknown $u_{\varepsilon}(t, x)$ is a function from $\mathbb{R}^{+} \times \mathbb{R}^{N}$ into $\mathbb{C}$. We assume that the coefficients $A(x, y)$ and $\rho(x, y)$ are real and sufficiently smooth bounded functions defined on $\mathbb{R}^{N} \times \mathbb{T}^{N}$, where $\mathbb{T}^{N}$ is the flat unit torus, i.e., the unit cell $(0,1)^{N}$ equipped with periodic boundary conditions (see Section 2 for more precise smoothness assumptions). The initial data $u_{\varepsilon}^{0}, u_{\varepsilon}^{1}$ are highly oscillating in resonance with the period $\varepsilon$. More precisely, they are given in terms of so-called Bloch waves $\psi_{n}(x, y, \xi) e^{2 i \pi \xi^{n} \cdot y}$ where $\psi_{n}$ is an eigenfunction, with corresponding eigenvalue $\lambda_{n}(x, \xi)$, of the Bloch spectral cell problem [9], [22]

$$
-\left(\operatorname{div}_{y}+2 \pi i \xi\right)\left(A(x, y)\left(\nabla_{y}+2 \pi i \xi\right) \psi_{n}\right)=\rho(x, y) \lambda_{n}(x, \xi) \psi_{n} \quad \text { in } \quad \mathbb{T}^{N}
$$

By standard arguments of spectral theory, (3) admits a countable sequence of real increasing eigenvalues $\left(\lambda_{n}\right)_{n \geq 1}$, repeating each value as many times as its multiplicity, with corresponding periodic eigenfunctions normalized in $L^{2}\left(\mathbb{T}^{N}\right)$ by

$$
\int_{\mathbb{T}^{N}} \rho(x, y)\left|\psi_{n}(x, y, \xi)\right|^{2} d y=1
$$

The Bloch parameter $\xi$ is usually interpreted as a reduced wave number and the square root of the eigenvalue is the time frequency defined by

$$
\omega_{n}(x, \xi)=\sqrt{\lambda_{n}(x, \xi)}
$$

Such Bloch wave initial data are called high frequency. In this context the homogenized limit of (1) can be studied by means of geometric optic method, also called WKB asymptotic expansion [9], [15] (this can be made rigorous by using the notion of semiclassical measures, or Wigner transforms [16], [17]). We shall not intend to give a full account of this theory here (we may also refer to section 6 in [4] for a brief review). Rather, we content ourselves by loosely stating that the asymptotic behavior of the solution $u_{\varepsilon}$ of (1) is given by the superposition of two waves, the amplitudes of which are solutions of Liouville transport equations, and the phases of which are the solutions of two eikonal equations. Solving these equations is somehow equivalent to solve, in the phase space $(x, \xi) \in \mathbb{R}^{N} \times \mathbb{T}^{N}$, the following two Hamiltonian systems

$$
\left\{\begin{array}{l}
\dot{x}=\nabla_{\xi}\left( \pm \omega_{n}(x, \xi)\right) \\
\dot{\xi}=-\nabla_{x}\left( \pm \omega_{n}(x, \xi)\right)
\end{array}\right.
$$

where the Hamiltonian $\pm \omega_{n}(x, \xi)$ is precisely the time frequency associated through (5) to the $n$th Bloch eigenvalue of (3).

In the case of a purely periodic medium, i.e., the coefficients $\rho$ and $A$ do not depend on $x$, the Hamiltonian systems (6) simplify considerably and the corresponding eikonal equations for the phases have explicit global solutions. In such a case, one can go beyond the geometric optic time scale and study long time dispersive effects for monochromatic initial data [4], [5] (see [13] for constant or smooth coefficients). Similar dispersive effects have also been described in the physics literature [19], [24]. 
In the present paper we stick to the case of locally periodic medium, i.e., the coefficients $\rho$ and $A$ depend both on $x$ and $y$. However, we focus on a special instance of initial data such that (6) has a trivial solution and a more precise analysis is required in order to describe the asymptotic behavior of (1). We consider initial data which are concentrating at a critical point $\left(x^{n}, \xi^{n}\right) \in \mathbb{R}^{N} \times \mathbb{T}^{N}$ in the phase space, i.e.,

$$
\nabla_{\xi} \lambda_{n}\left(x^{n}, \xi^{n}\right)=\nabla_{x} \lambda_{n}\left(x^{n}, \xi^{n}\right)=0,
$$

which, of course, implies the same for the Hamiltonian $\nabla_{\xi} \omega_{n}\left(x^{n}, \xi^{n}\right)=\nabla_{x} \omega_{n}\left(x^{n}, \xi^{n}\right)=0$. More precisely, for such a given critical point $\left(x^{n}, \xi^{n}\right)$ and for given functions $v^{0} \in H^{1}\left(\mathbb{R}^{N}\right)$ and $v^{1} \in H^{2}\left(\mathbb{R}^{N}\right)$, we choose initial data which are wave packets or coherent states, i.e.

$$
\begin{aligned}
& u_{\varepsilon}^{0}(x)=\psi_{n}\left(x^{n}, \frac{x}{\varepsilon}, \xi^{n}\right) e^{2 i \pi \frac{\xi^{n} \cdot x}{\varepsilon}} v^{0}\left(\frac{x-x^{n}}{\sqrt{\varepsilon}}\right) \\
& u_{\varepsilon}^{1}(x)=\frac{1}{\varepsilon} \psi_{n}\left(x^{n}, \frac{x}{\varepsilon}, \xi^{n}\right) e^{2 i \pi \frac{\xi^{n} \cdot x}{\varepsilon}} v^{1}\left(\frac{x-x^{n}}{\sqrt{\varepsilon}}\right)
\end{aligned}
$$

where the scale of focusing near $x^{n}$ is exactly $\sqrt{\varepsilon}$. For the critical point $\left(x^{n}, \xi^{n}\right)$ we use the notation $\omega_{n}=\sqrt{\lambda_{n}\left(x^{n}, \xi^{n}\right)}$.

Our first result (Theorems 3.1 and 3.2) shows that the solution of equation (1) is asymptotically given by

$$
u_{\varepsilon}(t, x) \approx \psi_{n}\left(x^{n}, \frac{x}{\varepsilon}, \xi^{n}\right) e^{2 i \pi \frac{\xi^{n} \cdot x}{\varepsilon}}\left(e^{i \frac{\omega_{n} t}{\varepsilon}} v^{+}\left(t, \frac{x-x^{n}}{\sqrt{\varepsilon}}\right)+e^{-i \frac{\omega_{n} t}{\varepsilon}} v^{-}\left(t, \frac{x-x^{n}}{\sqrt{\varepsilon}}\right)\right),
$$

where $v^{ \pm}(t, z)$ are the unique solutions of the two homogenized equations

$$
\begin{cases} \pm 2 i \frac{\partial v^{ \pm}}{\partial t}-\operatorname{div}\left(A^{*} \nabla v^{ \pm}\right)+\operatorname{div}\left(v^{ \pm} B^{*} z\right)+c^{*} v^{ \pm}+v^{ \pm} D^{*} z \cdot z=0 & \text { in } \mathbb{R}^{+} \times \mathbb{R}^{N} \\ v^{ \pm}(0, z)=\frac{1}{2}\left(v^{0}(z) \pm \frac{1}{i \omega_{n}} v^{1}(z)\right) & \text { in } \mathbb{R}^{N}\end{cases}
$$

where the tensorial coefficients are the full Hessian of the time frequency

$$
A^{*}=\frac{1}{8 \pi^{2}} \nabla_{\xi} \nabla_{\xi} \omega_{n}\left(x^{n}, \xi^{n}\right), B^{*}=\frac{1}{2 i \pi} \nabla_{\xi} \nabla_{x} \omega_{n}\left(x^{n}, \xi^{n}\right), D^{*}=\frac{1}{2} \nabla_{x} \nabla_{x} \omega_{n}\left(x^{n}, \xi^{n}\right),
$$

and the constant $c^{*}$ is defined by (25). In principle, a Schrödinger equation as (11), shows the dispersive nature of the envelope functions $v^{ \pm}$in the ansatz (10) (as explained in [4]). However, the presence of a quadratic potential and a "convective" term in (11) changes dramatically its interpretation.

Our second result is that, if the full Hessian of the Hamiltonian or time frequency $\nabla \nabla \omega_{n}\left(x^{n}, \xi^{n}\right)$ is positive definite (or negative definite), then the resolvent of (11) is compact, implying that (11) admits a countable family of eigenfunctions, with exponential decay at infinity and forming an orthonormal basis of $L^{2}\left(\mathbb{R}^{N}\right)$. In other words, any eigen-mode of (11) is localized in space. One can interpret this localization phenomenon by saying that the wave is "trapped" exponentially close to $x^{n}$. Physically, this effect is well-known and 
is effectively used for trapping light in perturbed photonic crystals [8] or fibers [23]. Our results are a partial explanation of this phenomenon since real photonic crystals feature line or point defects in periodic geometries while we consider instead smooth variations, with respect to $x$, of the coefficients of the wave equation.

A similar result was already explained in the context of quantum mechanics, more precisely for solid state physics where a Schrödinger equation with periodic coefficients describe the wave function of an electron in a periodic crystal [3] (see also [6] for the corresponding eigenvalue problem). There, localization is a well-known phenomenon which is called Anderson localization if the periodicity perturbation is random [7], [11]. Localization can also appear for classical waves (see [14] and reference therein). Usually, localization is obtained by introducing some randomness in a periodic media. One originality of our work is that localization is produced by a deterministic modulation of the periodic coefficients of the wave equation.

Our assumption on the existence of a critical point $\left(x^{n}, \xi^{n}\right)$ for the Hamiltonian, the Hessian of which is positive definite (or negative definite), is reasonable. It happens at least generically at the bottom or top of each Bloch band. On the other hand we do not require the existence of a strict gap, but just of a smooth critical point of the Hamiltonian.

Let us finish this discussion by emphasizing that we talk about "localization" only in the case of a positive definite (or negative definite) full Hessian of the Hamiltonian $\nabla \nabla \omega_{n}\left(x^{n}, \xi^{n}\right)$ which implies pure point spectrum of (11) with exponentially decaying eigenfunctions. In all other cases, the homogenized equation (11) for the envelope function has some essential spectrum and, therefore, no special property of localization of its eigenfunctions. In other words, our result of localization is Proposition 3.6 rather than Theorems 3.1 and 3.2. In our view, "localization" should not be confused with what we may call "concentration", which merely means that we can build approximate solutions of the wave equation (1) that have a support concentrating around a single point in the physical space. This latter phenomenon of "concentration" can be studied in a more general framework than that of Theorems 3.1 and 3.2: in particular, it may happen with purely periodic coefficients and can be checked by simple WKB or Wigner-measure arguments (it corresponds to a zero group velocity $\nabla_{\xi} \omega_{n}\left(x^{n}, \xi^{n}\right)=0$ and and a constant envelope function, as was shown in [17]). On the other hand, our type of "localization" can take place only with a macroscopic modulation of the periodic coefficients in (1) and yields a more precise asymptotic behavior of the envelope function than "concentration".

The content of our paper is as follows. Section 2 gives some basic properties of Bloch waves and two-scale convergence, as well as our main assumptions. Our main results are precisely stated in Section 3. Section 4 is devoted to the proof of the required a priori estimates. Sections 5 and 6 are concerned with the proofs of our convergence results.

\section{Preliminaries}

In this section we describe our notations, state our assumptions and give some preliminary results concerning the Bloch spectral problem. The coefficients $A(x, y)$ and $\rho(x, y)$ are real and uniformly bounded Carathéodory functions defined on $\mathbb{R}^{N} \times \mathbb{T}^{N}$, i.e., they belong to $L^{\infty}\left(\mathbb{T}^{N} ; C_{b}\left(\mathbb{R}^{N}\right)\right)$. The density is uniformly bounded from below by a positive constant, 
$\rho(x, y) \geq \rho_{0}>0$, and the matrix $A$ is symmetric and uniformly coercive, i.e., there exists $\nu>0$ such that $A(x, y) \zeta \cdot \zeta \geq \nu|\zeta|^{2}$ for any $\zeta \in \mathbb{R}^{N}$. Our main assumptions are as follows.

Hypothesis H1. There exist $x^{n} \in \mathbb{R}^{N}$ and $\xi^{n} \in \mathbb{T}^{N}$ such that

(i) $\lambda_{n}\left(x^{n}, \xi^{n}\right)$ is a simple eigenvalue,

(ii) $\left(x^{n}, \xi^{n}\right)$ is a critical point of $\lambda_{n}(x, \xi)$, i.e. $\nabla_{x} \lambda_{n}\left(x^{n}, \xi^{n}\right)=\nabla_{\xi} \lambda_{n}\left(x^{n}, \xi^{n}\right)=0$,

(iii) the eigenfunction $\psi_{n}\left(x^{n}, \cdot, \xi^{n}\right)$ belongs to $W^{1, \infty}\left(\mathbb{T}^{N}\right)$.

Hypothesis H2. The coefficients $A(x, y)$ and $\rho(x, y)$ are of class $C^{2}$ with respect to the variable $x$ in a neighborhood of $x=x^{n}$ and they admit the following second-order Taylor expansion

$A(x, y)=A\left(x_{n}, y\right)+\left(x-x_{n}\right) \cdot \nabla_{x} A\left(x_{n}, y\right)+\frac{1}{2}\left(x-x_{n}\right) \nabla_{x} \nabla_{x} A\left(x_{n}, y\right)\left(x-x_{n}\right)+o\left(\left|x-x_{n}\right|^{2}\right)$

and similarly for $\rho$.

We denote by $\nabla \nabla \lambda_{n}$ the full Hessian matrix of the function $\lambda_{n}(x, \xi)$ evaluated at the point $\left(x^{n}, \xi^{n}\right)$, i.e.

$$
\nabla \nabla \lambda_{n}=\left[\begin{array}{cc}
\nabla_{x} \nabla_{x} \lambda_{n} & \nabla_{\xi} \nabla_{x} \lambda_{n} \\
\nabla_{\xi} \nabla_{x} \lambda_{n} & \nabla_{\xi} \nabla_{\xi} \lambda_{n}
\end{array}\right]\left(x^{n}, \xi^{n}\right)
$$

Remark 2.1 Because of the simplicity assumption $\mathbf{H 1}(i)$ it is perfectly legitimate to differentiate the eigenvalue $\lambda_{n}$ as much as we need. Because of assumption $\mathbf{H 1}$ (ii) the Hessians of the eigenvalue $\lambda_{n}$ and of the time frequency $\omega_{n}$ are proportional at the point $\left(x^{n}, \xi^{n}\right)$, i.e., $2 \omega_{n} \nabla \nabla \omega_{n}=\nabla \nabla \lambda_{n}$.

Assumption $\mathbf{H 1}$ (iii) holds true, for example, if the coefficients $A(x, y)$ and $\rho(x, y)$ are piecewise smooth with respect to $y$.

For the sake of notational simplicity we define

$$
A_{0}(y):=A\left(x^{n}, y\right), \quad \lambda_{n}:=\lambda_{n}\left(x^{n}, \xi^{n}\right), \quad \psi_{n}(y):=\psi\left(x^{n}, y, \xi^{n}\right) \text { and } \rho_{0}(y)=\rho\left(x^{n}, y\right)
$$

and, similarly for the derivatives, we set

$$
A_{1, h}(y):=\frac{\partial A}{\partial x_{h}}\left(x^{n}, y\right), \quad A_{2, l h}(y):=\frac{\partial^{2} A}{\partial x_{l} \partial x_{h}}\left(x^{n}, y\right), \quad \text { for } l, h=1, \ldots, N .
$$

Analogous notation hold for all derivatives of $\rho, \psi_{n}$ and $\lambda_{n}$ with respect to the $x$-variables and the $\xi$-variables evaluated at $x=x^{n}$ and $\xi=\xi^{n}$.

Recall that we have three space variables, corresponding to three different scales, $x$, $y:=\left(x-x^{n}\right) / \varepsilon$ and $z:=\left(x-x^{n}\right) / \sqrt{\varepsilon}$. For any function $\theta(x, y)$ defined on $\mathbb{R}^{N} \times \mathbb{T}^{N}$, we define

$$
\theta^{\varepsilon}(x):=\theta\left(x, \frac{x}{\varepsilon}\right)=\theta\left(x^{n}+\sqrt{\varepsilon} z, \frac{z}{\sqrt{\varepsilon}}+\frac{x^{n}}{\varepsilon}\right):=\tilde{\theta}^{\varepsilon}(z)
$$


In what follows the symbols $\operatorname{div}_{y}$ and $\nabla_{y}$ are used to denote the divergence and gradient operators which act with respect to the $y$-variable while div and $\nabla$ will indicate the divergence and gradient operators which act with respect to the $x$ - or $z$-variable, according to the context.

We introduce the operator $\mathbb{A}_{n}(x, \xi)$ defined for $\psi \in L^{2}\left(\mathbb{T}^{N}\right)$ by

$$
\mathbb{A}_{n}(x, \xi) \psi:=-\left(\operatorname{div}_{y}+2 i \pi \xi\right)\left(A(x, y)\left(\nabla_{y}+2 i \pi \xi\right) \psi\right)-\lambda_{n}(x, \xi) \rho(x, y) \psi .
$$

Under assumptions $\mathbf{H 1}$ and $\mathbf{H 2}$ we can differentiate the Bloch spectral equation (3) in a neighborhood of the point $\left(x^{n}, \xi^{n}\right)$ in the phase space [18]. Denoting by $\left(e_{k}\right)_{1 \leq k \leq N}$ the canonical basis of $\mathbb{R}^{N}$, the first derivatives satisfy

$$
\mathbb{A}_{n}(x, \xi) \frac{\partial \psi_{n}}{\partial \xi_{k}}=2 \pi i e_{k} A\left(\nabla_{y}+2 \pi i \xi\right) \psi_{n}+\left(\operatorname{div}_{y}+2 \pi i \xi\right)\left(A 2 \pi i e_{k} \psi_{n}\right)+\rho \frac{\partial \lambda_{n}}{\partial \xi_{k}} \psi_{n}
$$

and

$$
\mathbb{A}_{n}(x, \xi) \frac{\partial \psi_{n}}{\partial x_{l}}=\left(\operatorname{div}_{y}+2 \pi i \xi\right)\left(\frac{\partial A}{\partial x_{l}}\left(\nabla_{y}+2 \pi i \xi\right) \psi_{n}\right)+\frac{\partial \rho}{\partial x_{l}} \lambda_{n} \psi_{n}+\rho \frac{\partial \lambda_{n}}{\partial x_{l}} \psi_{n}
$$

In the same manner we can obtain formulas for the second order derivatives, namely

$$
\begin{aligned}
\mathbb{A}_{n}(x, \xi) \frac{\partial^{2} \psi_{n}}{\partial x_{h} \partial \xi_{k}}=\quad & \left(\operatorname{div}_{y}+2 \pi i \xi\right) \frac{\partial A}{\partial x_{h}}\left(\nabla_{y}+2 \pi i \xi\right) \frac{\partial \psi_{n}}{\partial \xi_{k}} \\
+ & 2 \pi i e_{k} \frac{\partial A}{\partial x_{h}}\left(\nabla_{y}+2 \pi i \xi\right) \psi_{n}+\left(\operatorname{div}_{y}+2 \pi i \xi\right)\left(\frac{\partial A}{\partial x_{h}} 2 \pi i e_{k} \psi_{n}\right) \\
+ & 2 \pi i e_{k} A\left(\nabla_{y}+2 \pi i \xi\right) \frac{\partial \psi_{n}}{\partial x_{h}}+\left(\operatorname{div}_{y}+2 \pi i \xi\right)\left(A 2 \pi i e_{k} \frac{\partial \psi_{n}}{\partial x_{h}}\right) \\
+ & \frac{\partial \rho}{\partial x_{h}} \lambda_{n} \frac{\partial \psi_{n}}{\partial \xi_{k}}+\rho \frac{\partial \lambda_{n}}{\partial x_{h}} \frac{\partial \psi_{n}}{\partial \xi_{k}}+\frac{\partial \rho}{\partial x_{h}} \frac{\partial \lambda_{n}}{\partial \xi_{k}} \psi_{n}+\rho \frac{\partial^{2} \lambda_{n}}{\partial x_{h} \partial \xi_{k}} \psi_{n}+\rho \frac{\partial \lambda_{n}}{\partial \xi_{k}} \frac{\partial \psi_{n}}{\partial x_{h}}, \\
\mathbb{A}_{n}(x, \xi) \frac{\partial^{2} \psi_{n}}{\partial x_{h} \partial x_{l}=\quad}= & \left.\operatorname{div}_{y}+2 \pi i \xi\right) \frac{\partial^{2} A}{\partial x_{h} \partial x_{l}}\left(\nabla_{y}+2 \pi i \xi\right) \psi_{n}+\frac{\partial^{2} \rho}{\partial x_{h} \partial x_{l}} \lambda_{n} \psi_{n}+\rho \frac{\partial^{2} \lambda_{n}}{\partial x_{h} \partial x_{l}} \psi_{n} \\
+ & \left(\operatorname{div}_{y}+2 \pi i \xi\right) \frac{\partial A}{\partial x_{h}}\left(\nabla_{y}+2 \pi i \xi\right) \frac{\partial \psi_{n}}{\partial x_{l}}+\left(\operatorname{div}_{y}+2 \pi i \xi\right) \frac{\partial A}{\partial x_{l}}\left(\nabla_{y}+2 \pi i \xi\right) \frac{\partial \psi_{n}}{\partial x_{h}} \\
+ & \frac{\partial \rho}{\partial x_{h}} \lambda_{n} \frac{\partial \psi_{n}}{\partial x_{l}}+\frac{\partial \rho}{\partial x_{l}} \lambda_{n} \frac{\partial \psi_{n}}{\partial x_{h}}+\rho \frac{\partial \lambda_{n}}{\partial x_{h}} \frac{\partial \psi_{n}}{\partial x_{l}}+\rho \frac{\partial \lambda_{n}}{\partial x_{l}} \frac{\partial \psi_{n}}{\partial x_{h}}+\frac{\partial \rho}{\partial x_{l}} \frac{\partial \lambda_{n}}{\partial x_{h}} \psi_{n}+\frac{\partial \rho}{\partial x_{h}} \frac{\partial \lambda_{n}}{\partial x_{l}} \psi_{n}, \\
\mathbb{A}_{n}(x, \xi) \frac{\partial^{2} \psi_{n}}{\partial \xi_{l} \partial \xi_{k}}=\quad & 2 \pi i e_{l} A\left(\nabla_{y}+2 \pi i \xi\right) \frac{\partial \psi_{n}}{\partial \xi_{k}}+\left(\operatorname{div}_{y}+2 \pi i \xi\right) A 2 \pi i e_{l} \frac{\partial \psi_{n}}{\partial \xi_{k}} \\
+ & 2 \pi i e_{k} A\left(\nabla_{y}+2 \pi i \xi\right) \frac{\partial \psi_{n}}{\partial \xi_{l}}+\left(\operatorname{div}_{y}+2 \pi i \xi\right) A 2 \pi i e_{k} \frac{\partial \psi_{n}}{\partial \xi_{l}} \\
+ & \rho \frac{\partial \lambda_{n}}{\partial \xi_{l}} \frac{\partial \psi_{n}}{\partial \xi_{k}}+\rho \frac{\partial \lambda_{n}}{\partial \xi_{k}} \frac{\partial \psi_{n}}{\partial \xi_{l}}-4 \pi^{2} e_{k} A e_{l} \psi_{n}-4 \pi^{2} e_{l} A e_{k} \psi_{n}+\rho \frac{\partial^{2} \lambda_{n}}{\partial \xi_{l} \partial \xi_{k}} \psi_{n} .
\end{aligned}
$$

By integrating these equations for the second order derivatives against $\psi_{n}$, recalling the normalization (4) of the eigenfunctions and taking $x=x^{n}$, we obtain the following formulas that will be useful in the sequel.

Lemma 2.2 Under assumptions $\mathbf{H 1}$ and $\mathbf{H 2}$ the following equalities hold:

$$
\begin{aligned}
& \int_{\mathbb{T}^{N}} \frac{1}{2 \pi i}\left[A_{1, h}\left(\nabla_{y}+2 \pi i \xi^{n}\right) \frac{\partial \psi_{n}}{\partial \xi_{k}} \cdot\left(\nabla_{y}-2 \pi i \xi^{n}\right) \bar{\psi}_{n}-\rho_{1, h} \lambda_{n} \frac{\partial \psi_{n}}{\partial \xi_{k}} \bar{\psi}_{n}\right] d y \\
& +\int_{\mathbb{T}^{N}}\left[A_{1, h} e_{k} \psi_{n}\left(\nabla_{y}-2 \pi i \xi^{n}\right) \bar{\psi}_{n}+A_{0} e_{k} \frac{\partial \psi_{n}}{\partial x_{h}} \cdot\left(\nabla_{y}-2 \pi i \xi^{n}\right) \bar{\psi}_{n}\right] d y \\
& -\int_{\mathbb{T}^{N}}\left[e_{k} \bar{\psi}_{n} A_{1, h}\left(\nabla_{y}+2 \pi i \xi^{n}\right) \psi_{n}+e_{k} \bar{\psi}_{n} A_{0}\left(\nabla_{y}+2 \pi i \xi^{n}\right) \frac{\partial \psi_{n}}{\partial x_{h}}\right] d y \\
& -\frac{1}{2 \pi i} \frac{\partial^{2} \lambda_{n}}{\partial x_{h} \partial \xi_{k}}=0
\end{aligned}
$$




$$
\begin{aligned}
& \int_{\mathbb{T}^{N}}\left[A_{2, l h}\left(\nabla_{y}+2 \pi i \xi^{n}\right) \psi_{n} \cdot\left(\nabla_{y}-2 \pi i \xi^{n}\right) \bar{\psi}_{n}-\left(\rho_{2, l h} \lambda_{n}+\rho \frac{\partial^{2} \lambda_{n}}{\partial x_{h} \partial x_{l}}\right)\left|\psi_{n}\right|^{2}\right] d y \\
& +\int_{\mathbb{T}^{N}}\left[A_{1, h}\left(\nabla_{y}+2 \pi i \xi^{n}\right) \frac{\partial \psi_{n}}{\partial x_{l}} \cdot\left(\nabla_{y}-2 \pi i \xi^{n}\right) \bar{\psi}_{n}-\rho_{1, h} \lambda_{n} \frac{\partial \psi_{n}}{\partial x_{l}} \bar{\psi}_{n}\right] d y \\
& +\int_{\mathbb{T}^{N}}\left[A_{1, l}\left(\nabla_{y}+2 \pi i \xi^{n}\right) \frac{\partial \psi_{n}}{\partial x_{h}} \cdot\left(\nabla_{y}-2 \pi i \xi^{n}\right) \bar{\psi}_{n}-\rho_{1, l} \lambda_{n} \frac{\partial \psi_{n}}{\partial x_{h}} \bar{\psi}_{n}\right] d y=0 \\
& \int_{\mathbb{T}^{N}}\left[2 \pi i e_{k} A_{0}\left(\nabla_{y}+2 \pi i \xi^{n}\right) \frac{\partial \psi_{n}}{\partial \xi_{l}} \bar{\psi}_{n}-\left(A_{0} 2 \pi i e_{k} \frac{\partial \psi_{n}}{\partial \xi_{l}}\right)\left(\nabla_{y}-2 \pi i \xi^{n}\right) \bar{\psi}_{n}\right] d y \\
& +\int_{\mathbb{T}^{N}}\left[2 \pi i e_{l} A_{0}\left(\nabla_{y}+2 \pi i \xi^{n}\right) \frac{\partial \psi_{n}}{\partial \xi_{k}} \bar{\psi}_{n}-\left(A_{0} 2 \pi i e_{l} \frac{\partial \psi_{n}}{\partial \xi_{k}}\right)\left(\nabla_{y}-2 \pi i \xi^{n}\right) \bar{\psi}_{n}\right] d y \\
& -\int_{\mathbb{T}^{N}}\left[4 \pi^{2} e_{k} A_{0} e_{l}\left|\psi_{n}\right|^{2}+4 \pi^{2} e_{l} A_{0} e_{k}\left|\psi_{n}\right|^{2}\right] d y \\
& +\frac{\partial^{2} \lambda_{n}}{\partial \xi_{l} \partial \xi_{k}}=0
\end{aligned}
$$

We also recall the variational formulations of $\psi_{n}^{\varepsilon}(x)=\psi_{n}\left(x^{n}, \frac{x}{\varepsilon}, \xi^{n}\right)$ and of its derivatives.

Lemma 2.3 Let $\varphi(z)$ be a smooth compactly supported function defined from $\mathbb{R}^{N}$ into $\mathbb{C}$. Under assumptions $\mathbf{H 1}$ and $\mathbf{H} \mathbf{2}$ the following equalities hold:

$$
\begin{gathered}
\int_{\mathbb{R}^{N}}\left[A_{0}^{\varepsilon}\left(\nabla_{y}+2 \pi i \xi^{n}\right) \psi_{n}^{\varepsilon} \cdot\left(\sqrt{\varepsilon} \nabla-2 \pi i \xi^{n}\right) \bar{\varphi}(z)-\rho_{0}^{\varepsilon} \lambda_{n}^{\varepsilon} \psi_{n}^{\varepsilon} \bar{\varphi}(z)\right] d z=0, \\
\int_{\mathbb{R}^{N}}\left[A_{0}^{\varepsilon}\left(\nabla_{y}+2 \pi i \xi^{n}\right) \frac{\partial \psi_{n}^{\varepsilon}}{\partial \xi_{k}} \cdot\left(\sqrt{\varepsilon} \nabla-2 \pi i \xi^{n}\right) \bar{\varphi}(z)-\rho_{0}^{\varepsilon} \lambda_{n}^{\varepsilon} \frac{\partial \psi_{n}^{\varepsilon}}{\partial \xi_{k}} \bar{\varphi}(z)\right] d z \\
+\int_{\mathbb{R}^{N}}\left[-2 \pi i e_{k} A_{0}^{\varepsilon}\left(\nabla_{y}+2 \pi i \xi^{n}\right) \psi_{n}^{\varepsilon} \bar{\varphi}(z)+A_{0}^{\varepsilon} 2 \pi i e_{k} \psi_{n}^{\varepsilon}\left(\sqrt{\varepsilon} \nabla-2 \pi i \xi^{n}\right) \bar{\varphi}(z)\right] d z=0 \\
\int_{\mathbb{R}^{N}}\left[A_{0}^{\varepsilon}\left(\nabla_{y}+2 \pi i \xi^{n}\right) \frac{\partial \psi_{n}^{\varepsilon}}{\partial x_{l}} \cdot\left(\sqrt{\varepsilon} \nabla-2 \pi i \xi^{n}\right) \bar{\varphi}(z)-\rho_{0}^{\varepsilon} \lambda_{n}^{\varepsilon} \frac{\partial \psi_{n}^{\varepsilon}}{\partial x_{l}} \bar{\varphi}(z)\right] d z \\
+\int_{\mathbb{R}^{N}}\left[A_{1, l}^{\varepsilon}\left(\nabla_{y}+2 \pi i \xi^{n}\right) \psi_{n}^{\varepsilon} \cdot\left(\sqrt{\varepsilon} \nabla-2 \pi i \xi^{n}\right) \bar{\varphi}(z)-\rho_{1, l}^{\varepsilon} \lambda_{n}^{\varepsilon} \psi_{n}^{\varepsilon} \bar{\varphi}(z)\right] d z=0 .
\end{gathered}
$$

We recall the notion of two-scale convergence [1], [20] with a small parameter $\delta>0$ which will be equal to $\sqrt{\epsilon}$ in the sequel.

Proposition 2.4 Let $f_{\delta}$ be a sequence uniformly bounded in $L^{2}\left(\mathbb{R}^{N}\right)$.

(1) There exists a subsequence, still denoted by $f_{\delta}$, and a limit $f_{0}(x, y) \in L^{2}\left(\mathbb{R}^{N} \times \mathbb{T}^{N}\right)$ such that $f_{\delta}$ two-scale converges weakly to $f_{0}$ in the sense that

$$
\lim _{\delta \rightarrow 0} \int_{\mathbb{R}^{N}} f_{\delta}(x) \phi(x, x / \delta) d x=\int_{\mathbb{R}^{N}} \int_{\mathbb{T}^{N}} f_{0}(x, y) \phi(x, y) d x d y
$$

for all functions $\phi(x, y) \in L^{2}\left(\mathbb{R}^{N} ; C\left(\mathbb{T}^{N}\right)\right)$. 
(2) Assume further that $f_{\delta}$ two-scale converges weakly to $f_{0}$ and that

$$
\lim _{\delta \rightarrow 0}\left\|f_{\delta}\right\|_{L^{2}\left(\mathbb{R}^{N}\right)}=\left\|f_{0}\right\|_{L^{2}\left(\mathbb{R}^{N} \times \mathbb{T}^{N}\right)} .
$$

Then $f_{\delta}$ is said to two-scale converges strongly to its limit $f_{0}$ in the sense that, if $f_{0}$ is smooth enough, e.g. $f_{0}(x, y) \in L^{2}\left(\mathbb{R}^{N} ; C\left(\mathbb{T}^{N}\right)\right)$, we have

$$
\lim _{\delta \rightarrow 0} \int_{\mathbb{R}^{N}}\left|f_{\delta}(x)-f_{0}(x, x / \delta)\right|^{2} d x=0
$$

(3) Assume that $\delta \nabla f_{\delta}$ is also uniformly bounded in $L^{2}\left(\mathbb{R}^{N}\right)^{N}$. Then there exists a subsequence, still denoted by $f_{\delta}$, and a limit $f_{0}(x, y) \in L^{2}\left(\mathbb{R}^{N} ; H^{1}\left(\mathbb{T}^{N}\right)\right)$ such that $f_{\delta}$ two-scale converges weakly to $f_{0}(x, y)$ and $\delta \nabla f_{\delta}$ two-scale converges weakly to $\nabla_{y} f_{0}(x, y)$.

\section{Main Results}

In order to apply the two-scale convergence of Proposition 2.4, we first need to remove the oscillating phase in $u_{\varepsilon}$ and rescale it to the concentration scale $\sqrt{\varepsilon}$. This is the purpose of the changes of unknowns (23) and (26) which are necessary to pass to the limit and get the homogenized equations.

Theorem 3.1 Assume that $\mathbf{H 1}$ and $\mathbf{H 2}$ hold true and that the initial datas $u_{\varepsilon}^{0}$ and $u_{\varepsilon}^{1}$ are of the form (8) and (9), respectively, with $v^{0} \in H^{1}\left(\mathbb{R}^{N}\right)$ and $v^{1} \in H^{2}\left(\mathbb{R}^{N}\right)$. Define

$$
v_{\varepsilon}^{+}\left(t, \frac{x-x^{n}}{\sqrt{\varepsilon}}\right)=u_{\varepsilon}(t, x) e^{-i \frac{\omega_{n} t}{\varepsilon}} e^{-2 i \pi \frac{\xi^{n} \cdot x}{\varepsilon}}
$$

where $u_{\varepsilon}$ is the solution of (1). Then $v_{\varepsilon}^{+}(t, z)$ two-scale converges weakly to $\psi_{n}(y) v^{+}(t, z)$ and $v^{+}$is the unique solution of the homogenized Schrödinger equation

$$
\begin{cases}2 i \frac{\partial v^{+}}{\partial t}-\operatorname{div}\left(A^{*} \nabla v^{+}\right)+\operatorname{div}\left(v^{+} B^{*} z\right)+c^{*} v^{+}+v^{+} D^{*} z \cdot z=0 & \text { in } \mathbb{R}^{N} \times \mathbb{R}^{+} \\ v^{+}(0, z)=\frac{1}{2}\left(v^{0}(z)+\frac{1}{i \omega_{n}} v^{1}(z)\right) & \text { in } \mathbb{R}^{N}\end{cases}
$$

where

$$
A^{*}=\frac{1}{8 \pi^{2}} \nabla_{\xi} \nabla_{\xi} \omega_{n}\left(x^{n}, \xi^{n}\right), B^{*}=\frac{1}{2 i \pi} \nabla_{\xi} \nabla_{x} \omega_{n}\left(x^{n}, \xi^{n}\right), D^{*}=\frac{1}{2} \nabla_{x} \nabla_{x} \omega_{n}\left(x^{n}, \xi^{n}\right)
$$

and $c^{*}$ is given by

$c^{*}=\int_{\mathbb{T}^{N}}\left[A\left(\nabla_{y}+2 i \pi \xi^{n}\right) \psi_{n} \cdot \frac{\partial \bar{\psi}_{n}}{\partial x_{k}} e_{k}-A\left(\nabla_{y}-2 i \pi \xi^{n}\right) \frac{\partial \bar{\psi}_{n}}{\partial x_{k}} \cdot \psi_{n} e_{k}-A_{1, k}\left(\nabla_{y}-2 i \pi \xi^{n}\right) \bar{\psi}_{n} \cdot \psi_{n} e_{k}\right] d y \cdot(25$

A similar result holds true for

$$
v_{\varepsilon}^{-}\left(t, \frac{x-x^{n}}{\sqrt{\varepsilon}}\right)=u_{\varepsilon}(t, x) e^{i \frac{\omega_{n} t}{\varepsilon}} e^{-2 i \pi \frac{\xi^{n} \cdot x}{\varepsilon}},
$$


namely, $v_{\varepsilon}^{-}(t, z)$ two-scale converges weakly to $\psi_{n}(y) v^{-}(t, z)$, where $v^{-}$is the unique solution of the homogenized Schrödinger equation

$$
\begin{cases}-2 i \frac{\partial v^{-}}{\partial t}-\operatorname{div}\left(A^{*} \nabla v^{-}\right)+\operatorname{div}\left(v^{-} B^{*} z\right)+c^{*} v^{-}+v^{-} D^{*} z \cdot z=0 & \text { in } \mathbb{R}^{N} \times \mathbb{R}^{+} \\ v^{-}(0, z)=\frac{1}{2}\left(v^{0}(z)-\frac{1}{i \omega_{n}} v^{1}(z)\right) & \text { in } \mathbb{R}^{N}\end{cases}
$$

Remark that, if $v^{0}$ and $v^{1}$ are real-valued functions, then we deduce that $v^{-}=\overline{v^{+}}$. Theorem 3.1 gives two different limit behaviors for $u_{\varepsilon}$, according to the two different phases in (23) and (26). However each of these limits carry only half of the initial data. The next result explains that the sum of these two waves is a valid approximation of the solution $u_{\varepsilon}$ of (1). In other words we now state a strong two-scale convergence result instead of a weak one as in Theorem 3.1.

Theorem 3.2 Assume that $\mathbf{H 1}$ and $\mathbf{H 2}$ hold true and that $v^{0} \in H^{2}\left(\mathbb{R}^{N}\right)$ and $v^{1} \in H^{2}\left(\mathbb{R}^{N}\right)$. If $\xi^{n}=0$, assume furthermore that

$$
\left\{\begin{array}{l}
v^{1} \in L^{1}\left(\mathbb{R}^{N}\right) \text { if } N \geq 3 \\
\int_{\mathbb{R}^{N}} v^{1}(z) d z=0 \text { and } \mathcal{F} v^{1} \in C^{0, \alpha}\left(B_{0}\right) \text { with } \alpha>1-N / 2 \text { if } N \leq 2 .
\end{array}\right.
$$

where $\mathcal{F} v^{1}(\xi)$ denotes the Fourier transform of $v^{1}(x)$ and $B_{0}$ is a small open ball around the origin. Define the ansatz

$$
u_{\varepsilon}^{\text {approx }}(t, x)=\psi_{n}\left(x^{n}, \frac{x}{\varepsilon}, \xi^{n}\right) e^{2 i \pi \frac{\xi^{n} \cdot x}{\varepsilon}}\left(e^{i \frac{\omega_{n} t}{\varepsilon}} v^{+}\left(t, \frac{x-x^{n}}{\sqrt{\varepsilon}}\right)+e^{-i \frac{\omega_{n} t}{\varepsilon}} v^{-}\left(t, \frac{x-x^{n}}{\sqrt{\varepsilon}}\right)\right)
$$

where $v^{ \pm}(t, z)$ are the solutions of the two homogenized equations (24) and (27). Then it satisfies

$$
\lim _{\varepsilon \rightarrow 0} \frac{\left\|u_{\varepsilon}(t, x)-u_{\varepsilon}^{\text {approx }}(t, x)\right\|_{L^{2}\left((0, T) \times \mathbb{R}^{N}\right)}}{\left\|u_{\varepsilon}^{\text {approx }}(t, x)\right\|_{L^{2}\left((0, T) \times \mathbb{R}^{N}\right)}}=0
$$

for any final time $T>0$.

Remark 3.3 Theorem 3.2 gives a relative error going to zero. Indeed, it is easily shown, upon rescaling at scale $\sqrt{\varepsilon}$, that $\left\|u_{\varepsilon}^{\text {approx }}(t, x)\right\|_{L^{2}\left((0, T) \times R^{N}\right)}$ is of order $\varepsilon^{N / 4}$. Actually this estimate for $u_{\varepsilon}^{\text {approx }}$ is valid in $L^{2}\left(\mathbb{R}^{N}\right)$ for almost every time $t$. However, the error estimate requires a time integration and is not valid for almost every time $t$.

Remark 3.4 Assumption (28) is technical and is used merely in the a priori estimate of Lemma 6.2. We do not know if it is absolutely necessary or not. However the assumption that $v^{1}$ has zero average is reminiscent of a similar assumption used to prove that the solution of the wave equation in a periodic domain is uniformly bounded in $L^{\infty}\left(\mathbb{R}^{+}: L^{2}\left(\mathbb{R}^{N}\right)\right.$ ) (see for example Section 2.3.2 in [2]). 
Before we prove Theorems 3.1 and 3.2 let us analyze the homogenized Schrödinger equation (24). We define the following unbounded operator acting in $L^{2}\left(\mathbb{R}^{N}\right)$

$$
\mathbb{A}^{*} \phi:=-\operatorname{div}\left(A^{*} \nabla \phi\right)+\operatorname{div}\left(\phi B^{*} z\right)+c^{*} \phi+\phi D^{*} z \cdot z
$$

which already appears in the study of localization for the Schrödinger equation [3] (beware that the star symbol in (30) means "homogenized" and not adjoint). We show that (24) or equivalently (27) are well-posed.

Proposition 3.5 (Proposition 3.4 in [3]) The operator $\mathbb{A}^{*}$ defined in (30) is essentially self-adjoint. As a consequence, there exists a unique solution $v^{+}(t, z)$ of $(24)$ in $C\left(\mathbb{R}^{+} ; L^{2}\left(\mathbb{R}^{N}\right)\right)$. Furthermore, it satisfies the energy conservation

$$
\left\|v^{+}(t, \cdot)\right\|_{L^{2}\left(\mathbb{R}^{N}\right)}=\left\|v^{+}(0, \cdot)\right\|_{L^{2}\left(\mathbb{R}^{N}\right)} \quad \forall t \in \mathbb{R}^{+} .
$$

Proof. We thank the anonymous referee to point out a flaw in the original proof of this result in [3] where we ignored the fact that the domain of the unbounded operator $\mathbb{A}^{*}$ may be different of that of its adjoint. We thus wrongly concluded that $\mathbb{A}^{*}$ is self-adjoint while we shall now merely prove that it is essentially self-adjoint, i.e. that its closure is self-adjoint. This last property is enough for our purpose since it implies that $\mathbb{A}^{*}$ has a unique self-adjoint extension. We briefly indicate how to modify the proof in [3]. First, a simple integration by parts shows that $\mathbb{A}^{*}$ is symmetric on $C_{0}^{\infty}\left(\mathbb{R}^{N}\right)$ because $\left(\frac{1}{2 i} \operatorname{tr} B^{*}+\operatorname{Im} c^{*}\right)=0$. This last identity is obtained by a combination of a formula for $c^{*}$, deduced from (15) multiplied by $\frac{\partial \bar{\psi}_{n}}{\partial x_{k}}$, and another formula for $\nabla_{\xi} \nabla_{x} \lambda_{n}$, and thus for $B^{*}$, deduced from (17) where we plug (15) multiplied by $\frac{\partial \bar{\psi}_{n}}{\partial x_{h}}$. Second, we use Theorem X.37, page 197 in volume II of [22], to prove that $\mathbb{A}^{*}$ is essentially self-adjoint. Introducing the self-adjoint operator $N=-\Delta+|x|^{2}+1$, with domain $H^{2}\left(\mathbb{R}^{N}\right) \cap L^{2}\left(\mathbb{R}^{N},|x|^{2} d x\right)$, we easily check the assumptions of this theorem, namely that there exists a constant $C>0$ such that, for any $\phi \in C_{0}^{\infty}\left(\mathbb{R}^{N}\right)$,

$$
\left\|\mathbb{A}^{*} \phi\right\|_{L^{2}\left(\mathbb{R}^{N}\right)} \leq C\|N \phi\|_{L^{2}\left(\mathbb{R}^{N}\right)}
$$

and

$$
\left|\left\langle\mathbb{A}^{*} \phi, N \phi\right\rangle-\left\langle N \phi, \mathbb{A}^{*} \phi\right\rangle\right| \leq C\left\|N^{1 / 2} \phi\right\|_{L^{2}\left(\mathbb{R}^{N}\right)}^{2} .
$$

Third, by semigroup theory [10], [21], we deduce from the uniqueness of the self-adjoint extension of $\mathbb{A}^{*}$ that there exists a unique solution of $(24)$ in $C\left(\mathbb{R}^{+} ; L^{2}\left(\mathbb{R}^{N}\right)\right)$, which may not belong to $C\left(\mathbb{R}^{+} ; H^{1}\left(\mathbb{R}^{N}\right)\right)$ if the matrix $A^{*}$ is not positive definite or positive negative. The energy conservation for (24) is just a consequence of the symmetry of $\mathbb{A}^{*}$.

Eventually we recall a compactness result of [3] which is at the root of the localization phenomenon.

Proposition 3.6 (Proposition 3.5 in [3]) Assume that the matrix $\nabla \nabla \omega_{n}$ is positive definite (or equivalently positive negative). Then the resolvent of $\mathbb{A}^{*}$ is compact in $L^{2}\left(\mathbb{R}^{N}\right)$, and there exists an orthonormal basis $\left(\varphi_{n}\right)_{n \geq 1}$ of eigenfunctions of $\mathbb{A}^{*}$ which decay exponentially, i.e., for each $n$ there exists a constant $\gamma_{n}>0$ such that

$$
e^{\gamma_{n}|z|^{2}} \varphi_{n}(z), e^{\gamma_{n}|z|^{2}} \nabla \varphi_{n}(z) \in L^{2}\left(\mathbb{R}^{N}\right) .
$$


Remark 3.7 As we said in the introduction, we mean "localization" when the homogenized equations (24) and (27) for the two envelope functions have pure point spectrum with exponentially decaying eigenfunctions. Therefore, Proposition 3.6 is our result of localization rather than Theorems 3.1 and 3.2. In particular, what we call "localization" should not be confused with "concentration" which we define as the possibility of having sequences of solutions of the wave equation (1) concentrating around a single point in the physical space. This latter phenomenon is provided by Theorems 3.1 and 3.2 but it can be obtained in the simpler setting of purely periodic coefficients by means of well-known WKB or Wigner-measure arguments. In this latter setting, "concentration" means a zero group velocity $\nabla_{\xi} \omega_{n}\left(x^{n}, \xi^{n}\right)=0$ and and a constant envelope function: it was already derived in [17].

The proof of Theorem 3.1 relies on the change of unknowns (23): it is then possible to pass to the two-scale limit in the equation satisfied by $v_{\varepsilon}^{+}(t, z)$. We introduce the following notations

$$
\tilde{\rho}^{\varepsilon}(z)=\rho\left(x^{n}+\sqrt{\varepsilon} z, \frac{z}{\sqrt{\varepsilon}}+\frac{x^{n}}{\varepsilon}\right)
$$

and similar ones for $\tilde{A}^{\varepsilon}$ and $\tilde{\psi}_{n}^{\varepsilon}$. A simple computation yields

$$
\left\{\begin{array}{c}
\tilde{\rho}^{\varepsilon}\left(2 i \omega_{n} \frac{\partial v_{\varepsilon}^{+}}{\partial t}+\varepsilon \frac{\partial^{2} v_{\varepsilon}^{+}}{\partial t^{2}}\right)-\left(\operatorname{div}_{z}+\frac{2 i \pi \xi^{n}}{\sqrt{\varepsilon}}\right) \cdot\left(\tilde{A}^{\varepsilon}\left(\nabla_{z}+\frac{2 i \pi \xi^{n}}{\sqrt{\varepsilon}}\right) v_{\varepsilon}^{+}\right)=\tilde{\rho}^{\varepsilon} \frac{\lambda_{n}}{\varepsilon} v_{\varepsilon}^{+} \text {in } \mathbb{R}^{+} \times \mathbb{R}^{N} \\
v_{\varepsilon}^{+}(0)=\tilde{\psi}_{n}^{\varepsilon} v^{0} \text { in } \mathbb{R}^{N} \\
\frac{\partial v_{\varepsilon}^{+}}{\partial t}(0)=\frac{1}{\varepsilon} \tilde{\psi}_{n}^{\varepsilon}\left(-i \omega_{n} v^{0}+v^{1}\right) \text { in } \mathbb{R}^{N}
\end{array}\right.
$$

The solution $v_{\varepsilon}^{+}(t, z)$ of (34) satisfies a suitable a priori estimate for using the notion of two-scale convergence.

Lemma 3.8 For any final time $T>0$ there exists $C(T)>0$ independent of $\varepsilon$ such that the solution of (34) satisfies

$$
\begin{array}{r}
\left\|v_{\varepsilon}^{+}\right\|_{L^{\infty}\left((0, T) ; L^{2}\left(\mathbb{R}^{N}\right)\right)}+\varepsilon\left\|\frac{\partial v_{\varepsilon}^{+}}{\partial t}\right\|_{L^{\infty}\left((0, T) ; L^{2}\left(\mathbb{R}^{N}\right)\right)}+\sqrt{\varepsilon}\left\|\nabla_{z} v_{\varepsilon}^{+}\right\|_{L^{\infty}\left((0, T) ; L^{2}\left(\mathbb{R}^{N}\right)^{N}\right)} \\
\leq C(T)\left(\left\|v^{0}\right\|_{H^{1}\left(\mathbb{R}^{N}\right)}+\left\|v^{1}\right\|_{H^{2}\left(\mathbb{R}^{N}\right)}\right) .
\end{array}
$$

Notations. In the sequel we shall assume without loss of generality that $x^{n}=0$. This is always possible by a simple translation and it simplifies the writing of many formulas.

\section{A priori estimates (proof of Lemma 3.8)}

Although the statement of Lemma 3.8 involves $v_{\varepsilon}^{+}$, we shall prove an a priori estimate for $u_{\varepsilon}$, the solution of (1). Then, remarking that, by virtue of (23),

$\varepsilon^{N / 4}\left\|v_{\varepsilon}^{+}(t, \cdot)\right\|_{L^{2}\left(\mathbb{R}^{N}\right)}=\left\|u_{\varepsilon}(t, \cdot)\right\|_{L^{2}\left(\mathbb{R}^{N}\right)}, \quad \varepsilon^{N / 4}\left\|\varepsilon \frac{\partial v_{\varepsilon}^{+}}{\partial t}(t, \cdot)\right\|_{L^{2}\left(\mathbb{R}^{N}\right)}=\left\|\varepsilon \frac{\partial u_{\varepsilon}}{\partial t}(t, \cdot)-i \omega u_{\varepsilon}(t, \cdot)\right\|_{L^{2}\left(\mathbb{R}^{N}\right)}$, 
and

$$
\varepsilon^{N / 4}\left\|\sqrt{\varepsilon} \nabla_{z} v_{\varepsilon}^{+}(t, \cdot)\right\|_{L^{2}\left(\mathbb{R}^{N}\right)^{N}}=\left\|\varepsilon \nabla_{x} u_{\varepsilon}(t, \cdot)+2 i \pi \xi u_{\varepsilon}(t, \cdot)\right\|_{L^{2}\left(\mathbb{R}^{N}\right)^{N}},
$$

we easily deduce (35) from the corresponding estimates on $u_{\varepsilon}$.

In a first step we obtain the usual energy conservation by multiplying equation (1) by $\frac{\partial \bar{u}_{\varepsilon}}{\partial t}$ and integrating by parts

$$
\frac{d}{d t} E^{\varepsilon}(t)=0 \quad \text { with } \quad E^{\varepsilon}(t)=\frac{1}{2} \int_{\mathbb{R}^{N}}\left[\rho^{\varepsilon}\left|\frac{\partial u_{\varepsilon}}{\partial t}\right|^{2}+A^{\varepsilon} \nabla u_{\varepsilon} \cdot \nabla \overline{u_{\varepsilon}}\right] d x .
$$

Since the initial data $u_{\varepsilon}^{0}$ and $u_{\varepsilon}^{1}$ are defined by (8) and (9) respectively, and because $\psi_{n}$ belongs to $L^{\infty}\left(\mathbb{T}^{N}\right)$, we deduce

$$
\int_{\mathbb{R}^{N}} \rho^{\varepsilon}\left|\frac{\partial u_{\varepsilon}}{\partial t}(0)\right|^{2} d x=\frac{\varepsilon^{N / 2}}{\varepsilon^{2}} \int_{\mathbb{R}^{N}} \rho\left(\sqrt{\varepsilon} z, \frac{z}{\sqrt{\varepsilon}}\right)\left|\psi_{n}\left(\frac{z}{\sqrt{\varepsilon}}\right) v^{1}(z)\right|^{2} d z \leq C \varepsilon^{N / 2-2}\left\|v^{1}\right\|_{L^{2}\left(\mathbb{R}^{N}\right)^{2}}^{2} .
$$

A similar estimate holds for $\nabla u_{\varepsilon}(0)$ because assumption $\mathbf{H 1}$ (iii) tells us that $\nabla_{y} \psi_{n}$ belongs to $L^{\infty}\left(\mathbb{T}^{N}\right)^{N}$

$$
\int_{\mathbb{R}^{N}} A^{\varepsilon} \nabla u_{\varepsilon}(0) \cdot \nabla \overline{u_{\varepsilon}(0)} d x \leq C \varepsilon^{N / 2-2}\left(\left\|v^{0}\right\|_{L^{2}\left(\mathbb{R}^{N}\right)}^{2}+\varepsilon\left\|\nabla v^{0}\right\|_{L^{2}\left(\mathbb{R}^{N}\right)^{N}}^{2}\right),
$$

which implies

$$
E^{\varepsilon}(0) \leq C \varepsilon^{N / 2-2}\left(\left\|v^{0}\right\|_{H^{1}\left(\mathbb{R}^{N}\right)}^{2}+\left\|v^{1}\right\|_{L^{2}\left(\mathbb{R}^{N}\right)}^{2}\right) .
$$

This is only at this point that we use assumption $\mathbf{H 1}$ (iii) on the smoothness of $\psi_{n}$. Remark that we always have $\psi_{n} \in L^{\infty}\left(\mathbb{T}^{N}\right)$ by standard elliptic regularity and that we could have replaced $\mathbf{H 1}$ (iii) by an additional regularity of $v^{0}$ (using integration by parts as in the proof of Lemma 4.1 below). In any case we obtain

$$
\varepsilon\left\|\frac{\partial u_{\varepsilon}}{\partial t}\right\|_{L^{\infty}\left(\mathbb{R}^{+} ; L^{2}\left(\mathbb{R}^{N}\right)\right)}+\varepsilon\left\|\nabla u_{\varepsilon}\right\|_{L^{\infty}\left(\mathbb{R}^{+} ; L^{2}\left(\mathbb{R}^{N}\right)^{N}\right)} \leq C \varepsilon^{N / 4}\left(\left\|v^{0}\right\|_{H^{1}\left(\mathbb{R}^{N}\right)}+\left\|v^{1}\right\|_{L^{2}\left(\mathbb{R}^{N}\right)}\right) .
$$

In a second step we obtain an estimate for $u_{\varepsilon}$ in $L^{\infty}\left(\mathbb{R}^{+} ; L^{2}\left(\mathbb{R}^{N}\right)\right)$ following an argument of [4] based on a classical idea of time regularization. For a given $\alpha \neq 0$, we introduce a time primitive of $u_{\varepsilon}$ as

$$
\Psi_{\varepsilon}(x, t)=e^{-\alpha t}\left[\int_{0}^{t} e^{\alpha s} u_{\varepsilon}(x, s) d s+\chi_{\varepsilon}(x)\right]
$$

where $\chi_{\varepsilon}$ is defined as the unique solution in $H^{1}\left(\mathbb{R}^{N}\right)$ of the time-independent equation

$$
-\operatorname{div}\left(A\left(x, \frac{x}{\varepsilon}\right) \nabla \chi_{\varepsilon}\right)+\alpha^{2} \rho\left(x, \frac{x}{\varepsilon}\right) \chi_{\varepsilon}=\alpha \rho\left(x, \frac{x}{\varepsilon}\right) u_{\varepsilon}^{0}-\rho\left(x, \frac{x}{\varepsilon}\right) u_{\varepsilon}^{1} \quad \text { in } \mathbb{R}^{N} .
$$

A simple computation shows that $\Psi_{\varepsilon}$ satisfies

$$
\left\{\begin{aligned}
\rho\left(x, \frac{x}{\varepsilon}\right) \frac{\partial^{2} \Psi_{\varepsilon}}{\partial t^{2}}-\operatorname{div}\left(A\left(x, \frac{x}{\varepsilon}\right) \nabla \Psi_{\varepsilon}\right) & =0 & & \text { in } \mathbb{R}^{+} \times \mathbb{R}^{N} \\
\Psi_{\varepsilon}(0) & =\chi_{\varepsilon} & & \text { in } \mathbb{R}^{N} \\
\frac{\partial \Psi_{\varepsilon}}{\partial t}(0) & =u_{\varepsilon}^{0}-\alpha \chi_{\varepsilon} & & \text { in } \mathbb{R}^{N} .
\end{aligned}\right.
$$


The interest of (38) is that its initial data are one order smaller in $\varepsilon$ than those of (1) as stated in the following lemma.

Lemma 4.1 The solution of (37) satisfies

$$
\left\|\chi_{\varepsilon}\right\|_{H^{1}\left(\mathbb{R}^{N}\right)} \leq C \varepsilon^{N / 4}\left(\left\|v^{0}\right\|_{L^{2}\left(\mathbb{R}^{N}\right)}+\left\|v^{1}\right\|_{H^{2}\left(\mathbb{R}^{N}\right)}\right) .
$$

Postponing for a moment the proof of Lemma 4.1, we are now in a position to prove that

$$
\left\|u_{\varepsilon}\right\|_{L^{\infty}\left((0, T) ; L^{2}\left(\mathbb{R}^{N}\right)\right)} \leq C(T) \varepsilon^{N / 4}\left(\left\|v^{0}\right\|_{L^{2}\left(\mathbb{R}^{N}\right)}+\left\|v^{1}\right\|_{H^{2}\left(\mathbb{R}^{N}\right)}\right)
$$

which concludes the proof of Lemma 3.8. Indeed, since

$$
u_{\varepsilon}=\frac{\partial \Psi_{\varepsilon}}{\partial t}+\alpha \Psi_{\varepsilon}
$$

the standard energy conservation for (38), together with Lemma 4.1, implies that

$$
\left\|\frac{\partial \Psi_{\varepsilon}}{\partial t}\right\|_{L^{\infty}\left(\mathbb{R}^{+} ; L^{2}\left(\mathbb{R}^{N}\right)\right)} \leq C \varepsilon^{N / 4}\left(\left\|v^{0}\right\|_{L^{2}\left(\mathbb{R}^{N}\right)}+\left\|v^{1}\right\|_{H^{2}\left(\mathbb{R}^{N}\right)}\right) .
$$

Since $\Psi_{\varepsilon}(t)=\chi_{\varepsilon}+\int_{0}^{t} \frac{\partial \Psi_{\varepsilon}}{\partial t}(s) d s$, we deduce $(39)$.

Proof of Lemma 4.1. Without loss of generality we take $\alpha=1$. By multiplying (37) by $\bar{\chi}_{\varepsilon}$ we obtain

$$
\int_{\mathbb{R}^{N}}\left(A\left(x, \frac{x}{\varepsilon}\right) \nabla \chi_{\varepsilon} \cdot \nabla \overline{\chi_{\varepsilon}}+\rho\left(x, \frac{x}{\varepsilon}\right)\left|\chi_{\varepsilon}\right|^{2}\right) d x=\int_{\mathbb{R}^{N}} \rho\left(x, \frac{x}{\varepsilon}\right)\left(u_{\varepsilon}^{0}-u_{\varepsilon}^{1}\right) \overline{\chi_{\varepsilon}} d x .
$$

At first, we easily check that

$$
\int_{\mathbb{R}^{N}}\left|\rho^{\varepsilon} u_{\varepsilon}^{0} \overline{\chi_{\varepsilon}}\right| d x \leq C \varepsilon^{N / 4}\left\|v^{0}\right\|_{L^{2}\left(\mathbb{R}^{N}\right)}\left\|\chi_{\varepsilon}\right\|_{L^{2}\left(\mathbb{R}^{N}\right)}
$$

Since $u_{\varepsilon}^{1}=\varepsilon^{-1} \psi_{n}(x / \varepsilon) e^{2 i \pi \frac{\xi_{n} \cdot x}{\varepsilon}} v^{1}(x / \sqrt{\varepsilon})$ we define

$$
\Delta_{\varepsilon}:=\int_{\mathbb{R}^{N}} \rho\left(x, \frac{x}{\varepsilon}\right) \psi_{n}\left(\frac{x}{\varepsilon}\right) e^{2 i \pi \frac{\xi_{n} \cdot x}{\varepsilon}} v^{1}\left(\frac{x}{\sqrt{\varepsilon}}\right) \overline{\chi_{\varepsilon}}(x) d x .
$$

Let us now prove that

$$
\left|\Delta_{\varepsilon}\right| \leq C \varepsilon^{N / 4+1}\left\|v^{1}\right\|_{H^{2}\left(\mathbb{R}^{N}\right)}\left\|\chi_{\varepsilon}\right\|_{H^{1}\left(\mathbb{R}^{N}\right)}
$$

By Lemma 4.4 of [4] there exists a solution $\zeta \in C^{2}\left(\mathbb{R}^{N} ; C\left(\mathbb{T}^{N}\right)\right)^{N}$ of

$$
-\operatorname{div}_{y}\left(\zeta(x, y) e^{2 i \pi \xi^{n} \cdot y}\right)=\rho(x, y) \psi_{n}(y) e^{2 i \pi \xi^{n} \cdot y}
$$

Then

$$
\rho\left(x, \frac{x}{\varepsilon}\right) \psi_{n}\left(\frac{x}{\varepsilon}\right) e^{2 i \pi \frac{\xi^{n} \cdot x}{\varepsilon}}=-\varepsilon \operatorname{div}\left[\zeta\left(x, \frac{x}{\varepsilon}\right) e^{2 i \pi \frac{\xi^{n} \cdot x}{\varepsilon}}\right]+\varepsilon \operatorname{div}_{x} \zeta\left(x, \frac{x}{\varepsilon}\right) e^{2 i \pi \frac{\xi^{n} \cdot x}{\varepsilon}}
$$


Using $\zeta$ allows us to rewrite

$$
\begin{aligned}
\Delta_{\varepsilon} & =\varepsilon \int_{\mathbb{R}^{N}}\left[\zeta\left(x, \frac{x}{\varepsilon}\right) e^{2 i \pi \frac{\xi^{n} \cdot x}{\varepsilon}} \cdot\left(v^{1}\left(\frac{x}{\sqrt{\varepsilon}}\right) \nabla \bar{\chi}^{\varepsilon}(x)+\frac{1}{\sqrt{\varepsilon}} \bar{\chi}^{\varepsilon}(x) \nabla v^{1}\left(\frac{x}{\sqrt{\varepsilon}}\right)\right)\right] d x \\
& +\varepsilon \int_{\mathbb{R}^{N}} \operatorname{div}_{x} \zeta\left(x, \frac{x}{\varepsilon}\right) e^{2 i \pi \frac{\xi^{n} \cdot x}{\varepsilon}} v^{1}\left(\frac{x}{\sqrt{\varepsilon}}\right) \overline{\chi_{\varepsilon}}(x) d x .
\end{aligned}
$$

The first and last term in the right hand side of (42) are of order $\varepsilon$ as expected, but not the second term. We therefore perform another integration by parts using the solution $\theta \in C^{2}\left(\mathbb{R}^{N} ; C\left(\mathbb{T}^{N}\right)\right)^{N^{2}}$ of

$$
-\operatorname{div}_{y}\left(\theta(x, y) e^{2 i \pi \xi^{n} \cdot y}\right)=\zeta(x, y) e^{2 i \pi \xi^{n} \cdot y},
$$

given by another application of Lemma 4.4 of [4]. Thus we obtain

$$
\begin{aligned}
\sqrt{\varepsilon} \int_{\mathbb{R}^{N}} \zeta\left(x, \frac{x}{\varepsilon}\right) e^{2 i \pi \frac{\xi^{n} \cdot x}{\varepsilon}} \cdot \bar{\chi}^{\varepsilon}(x) \nabla v^{1}\left(\frac{x}{\sqrt{\varepsilon}}\right) d x & =\varepsilon^{3 / 2} \int_{\mathbb{R}^{N}} \theta\left(x, \frac{x}{\varepsilon}\right) e^{2 i \pi \frac{\xi^{n} \cdot x}{\varepsilon}} \cdot \nabla\left(\bar{\chi}^{\varepsilon} \nabla v^{1}\left(\frac{x}{\sqrt{\varepsilon}}\right)\right) d x \\
& +\varepsilon^{3 / 2} \int_{\mathbb{R}^{N}} \operatorname{div}_{x} \theta\left(x, \frac{x}{\varepsilon}\right) e^{2 i \pi \frac{\xi^{n} \cdot x}{\varepsilon}} \cdot\left(\bar{\chi}^{\varepsilon} \nabla v^{1}\left(\frac{x}{\sqrt{\varepsilon}}\right)\right) d x
\end{aligned}
$$

where all terms can be bounded by Cauchy-Schwarz inequality, using the fact that $\zeta$ and $\theta$ are bounded functions. It leads to the desired result (41). This completes the proof of the lemma.

\section{$5 \quad$ Weak convergence (proof of Theorem 3.1)}

For the sake of notational simplicity we now drop the notation , introduced in (33), which indicates a function of $z$ instead of $x$. In a first step we multiply equation (34) by a test function $\varepsilon \bar{\phi}^{\varepsilon}=\varepsilon \bar{\phi}\left(t, z, \frac{z}{\sqrt{\varepsilon}}\right)$, where $\phi(t, z, y)$ is a smooth, compactly supported, function defined on $\mathbb{R}^{+} \times \mathbb{R}^{N} \times \mathbb{T}^{N}$. Integrating by parts and using hypothesis $\mathbf{H} 2$ we obtain

$$
\begin{aligned}
& -2 i \omega_{n} \varepsilon \int_{0}^{+\infty} \int_{\mathbb{R}^{N}} \rho^{\varepsilon} v_{\varepsilon}^{+} \frac{\partial \bar{\phi}^{\varepsilon}}{\partial t} d z d t+\varepsilon^{2} \int_{0}^{+\infty} \int_{\mathbb{R}^{N}} \rho^{\varepsilon} v_{\varepsilon}^{+} \frac{\partial^{2} \bar{\phi}^{\varepsilon}}{\partial t^{2}} d z d t \\
& -2 i \omega_{n} \varepsilon \int_{\mathbb{R}^{N}} \rho^{\varepsilon} v_{\varepsilon}^{+}(0) \bar{\phi}^{\varepsilon}(0) d z-\varepsilon^{2} \int_{\mathbb{R}^{N}} \rho^{\varepsilon} v_{\varepsilon}^{+}(0) \frac{\partial \bar{\phi}^{\varepsilon}}{\partial t}(0) d z+\varepsilon^{2} \int_{\mathbb{R}^{N}} \rho^{\varepsilon} \frac{\partial v_{\varepsilon}^{+}}{\partial t}(0) \bar{\phi}^{\varepsilon}(0) d z \\
& +\int_{0}^{+\infty} \int_{\mathbb{R}^{N}}\left[A_{0}^{\varepsilon}+A_{1, h}^{\varepsilon} \sqrt{\varepsilon} z_{h}+\frac{1}{2} A_{2, l h}^{\varepsilon} \varepsilon z_{l} z_{h}+o(\varepsilon)\right]\left(\sqrt{\varepsilon} \nabla+2 i \pi \xi^{n}\right) v_{\varepsilon}^{+} \cdot\left(\sqrt{\varepsilon} \nabla-2 i \pi \xi^{n}\right) \bar{\phi}^{\varepsilon} d z d t \\
& -\int_{0}^{+\infty} \int_{\mathbb{R}^{N}}\left[\rho_{0}^{\varepsilon}+\rho_{1, h}^{\varepsilon} \sqrt{\varepsilon} z_{h}+\frac{1}{2} \rho_{2, l h}^{\varepsilon} \varepsilon z_{l} z_{h}+o(\varepsilon)\right] \lambda_{n} v_{\varepsilon}^{+} \bar{\phi}^{\varepsilon} d z d t=0 .
\end{aligned}
$$

Remark that the above $o(\varepsilon)$ holds true in the $L^{\infty}\left(\mathbb{R}^{N}\right)$-norm since the test function $\phi$ has compact support in $z$. From Proposition 2.4, and because of the a priori estimate (35), there exist a subsequence and a limit $v^{*}(t, z, y) \in L^{\infty}\left(\mathbb{R}^{+} ; L^{2}\left(\mathbb{R}^{N} ; H^{1}\left(\mathbb{T}^{N}\right)\right)\right)$ such that $v_{\varepsilon}^{+}(t, z)$ and 
$\sqrt{\varepsilon} \nabla v_{\varepsilon}^{+}(t, z)$ two-scale converge to $v^{*}$ and $\nabla_{y} v^{*}$ respectively. Passing to the two-scale limit in the above equality, we deduce a variational formulation for

$$
-\left(\operatorname{div}_{y}+2 i \pi \xi^{n}\right)\left(A_{0}(y)\left(\nabla_{y}+2 i \pi \xi^{n}\right) v^{*}\right)=\rho_{0} \lambda_{n} v^{*}
$$

By the simplicity of $\lambda_{n}$ (assumption H1), there exists a scalar function $v^{+}(t, z) \in L^{\infty}\left(\mathbb{R}^{+} ; L^{2}\left(\mathbb{R}^{N}\right)\right)$ such that

$$
v^{*}(t, z, y)=v^{+}(t, z) \psi_{n}(y)
$$

In a second step we multiply equation (34) by the complex conjugate of

$$
\Phi_{\varepsilon}(t, z)=\psi_{n}^{\varepsilon}(z) \phi(t, z)+\sqrt{\varepsilon} \sum_{k=1}^{N}\left(\frac{1}{2 \pi i} \frac{\partial \psi_{n}^{\varepsilon}}{\partial \xi_{k}} \frac{\partial \phi}{\partial z_{k}}+z_{k} \frac{\partial \psi_{n}^{\varepsilon}}{\partial x_{k}} \phi\right)
$$

where $\phi(t, z)$ is a smooth, compactly supported, function defined on $\mathbb{R}^{+} \times \mathbb{R}^{N}$. We decompose the resulting variational formulation in several pieces and pass to the limit in each of them separately.

The time derivative terms in (34) yield, first, by two successive integration by parts,

$$
\begin{aligned}
& \int_{0}^{+\infty} \int_{\mathbb{R}^{N}} \varepsilon \rho^{\varepsilon} \frac{\partial^{2} v_{\varepsilon}^{+}}{\partial t^{2}} \overline{\Phi^{\varepsilon}} d t d z= \\
& -\int_{0}^{+\infty} \int_{\mathbb{R}^{N}} \varepsilon \rho^{\varepsilon} \frac{\partial v_{\varepsilon}^{+}}{\partial t}\left[\overline{\psi_{n}^{\varepsilon}} \frac{\partial \bar{\phi}}{\partial t}+\sqrt{\varepsilon} \sum_{k=1}^{N}\left(-\frac{1}{2 \pi i} \frac{\partial \overline{\psi_{n}^{\varepsilon}}}{\partial \xi_{k}} \frac{\partial^{2} \bar{\phi}}{\partial t \partial z_{k}}+z_{k} \frac{\partial \overline{\psi_{n}^{\varepsilon}}}{\partial x_{k}} \frac{\partial \bar{\phi}}{\partial t}\right)\right] \\
& -\int_{\mathbb{R}^{N}} \varepsilon \rho^{\varepsilon} \frac{\partial v_{\varepsilon}^{+}}{\partial t}(0)\left[\overline{\psi_{n}^{\varepsilon} \phi}(0)+\sqrt{\varepsilon} \sum_{k=1}^{N}\left(-\frac{1}{2 \pi i} \frac{\partial \overline{\psi_{n}^{\varepsilon}}}{\partial \xi_{k}} \frac{\partial \bar{\phi}}{\partial z_{k}}(0)+z_{k} \frac{\partial \overline{\psi_{n}^{\varepsilon}}}{\partial x_{k}} \bar{\phi}(0)\right)\right]= \\
& +\int_{0}^{+\infty} \int_{\mathbb{R}^{N}} \varepsilon \rho^{\varepsilon} v_{\varepsilon}^{+}\left[\overline{\psi_{n}^{\varepsilon}} \frac{\partial^{2} \bar{\phi}}{\partial t^{2}}+\sqrt{\varepsilon} \sum_{k=1}^{N}\left(-\frac{1}{2 \pi i} \frac{\partial \overline{\psi_{n}^{\varepsilon}}}{\partial \xi_{k}} \frac{\partial^{3} \bar{\phi}}{\partial t^{2} \partial z_{k}}+z_{k} \frac{\partial \overline{\psi_{n}^{\varepsilon}}}{\partial x_{k}} \frac{\partial^{2} \bar{\phi}}{\partial t^{2}}\right)\right] \\
& \int_{\mathbb{R}^{N}} \varepsilon \rho^{\varepsilon} v_{\varepsilon}^{+}(0)\left[\overline{\psi_{n}^{\varepsilon}} \frac{\partial \bar{\phi}}{\partial t}(0)+\sqrt{\varepsilon} \sum_{k=1}^{N}\left(-\frac{1}{2 \pi i} \frac{\partial \overline{\psi_{n}^{\varepsilon}}}{\partial \xi_{k}} \frac{\partial^{2} \bar{\phi}}{\partial t \partial z_{k}}(0)+z_{k} \frac{\partial \overline{\psi_{n}^{\varepsilon}}}{\partial x_{k}} \frac{\partial \bar{\phi}}{\partial t}(0)\right)\right] \\
& -\int_{\mathbb{R}^{N}} \varepsilon \rho^{\varepsilon} \frac{\partial v_{\varepsilon}^{+}}{\partial t}(0)\left[\overline{\psi_{n}^{\varepsilon} \phi}(0)+\sqrt{\varepsilon} \sum_{k=1}^{N}\left(-\frac{1}{2 \pi i} \frac{\partial \overline{\psi_{n}^{\varepsilon}}}{\partial \xi_{k}} \frac{\partial \bar{\phi}}{\partial z_{k}}(0)+z_{k} \frac{\partial \overline{\psi_{n}^{\varepsilon}}}{\partial x_{k}} \bar{\phi}(0)\right)\right]= \\
& -\int_{\mathbb{R}^{N}} \rho^{\varepsilon}\left|\psi_{n}^{\varepsilon}\right|^{2}\left(-i \omega_{n} v^{0}+v^{1}\right) \bar{\phi}(0)+o(1)
\end{aligned}
$$


and second, by a single integration by parts,

$$
\begin{array}{r}
2 i \omega_{n} \int_{0}^{+\infty} \int_{\mathbb{R}^{N}} \rho^{\varepsilon} \frac{\partial v_{\varepsilon}^{+}}{\partial t} \overline{\Phi^{\varepsilon}} d t d z= \\
-2 i \omega_{n} \int_{0}^{+\infty} \int_{\mathbb{R}^{N}} \rho^{\varepsilon} v_{\varepsilon}^{+}\left[\bar{\psi}_{n}^{\varepsilon} \frac{\partial \bar{\phi}}{\partial t}+\sqrt{\varepsilon} \sum_{k=1}^{N}\left(-\frac{1}{2 \pi i} \frac{\partial \overline{\psi_{n}^{\varepsilon}}}{\partial \xi_{k}} \frac{\partial^{2} \bar{\phi}}{\partial t \partial z_{k}}+z_{k} \frac{\partial \overline{\psi_{n}^{\varepsilon}}}{\partial x_{k}} \frac{\partial \bar{\phi}}{\partial t}\right)\right] \\
-2 i \omega_{n} \int_{\mathbb{R}^{N}} \rho^{\varepsilon} v_{\varepsilon}^{+}(0)\left[\bar{\psi}_{n}^{\varepsilon}(0) \bar{\phi}+\sqrt{\varepsilon} \sum_{k=1}^{N}\left(-\frac{1}{2 \pi i} \frac{\partial \overline{\psi_{n}^{\varepsilon}}}{\partial \xi_{k}} \frac{\partial \bar{\phi}}{\partial z_{k}}(0)+z_{k} \frac{\partial \overline{\psi_{n}^{\varepsilon}}}{\partial x_{k}} \bar{\phi}(0)\right)\right]= \\
-2 i \omega_{n} \int_{0}^{+\infty} \int_{\mathbb{R}^{N}} \rho^{\varepsilon} v_{\varepsilon}^{+} \bar{\psi}_{n}^{\varepsilon} \frac{\partial \bar{\phi}}{\partial t}-2 i \omega_{n} \int_{\mathbb{R}^{N}} \rho^{\varepsilon}\left|\psi_{n}^{\varepsilon}\right|^{2} v^{0} \bar{\phi}(0)+o(1) .
\end{array}
$$

Passing to the two-scale limit in (43) plus (44) and recalling the normalization (4), we find

$$
-2 i \omega_{n} \int_{0}^{+\infty} \int_{\mathbb{R}^{N}} v^{+} \frac{\partial \bar{\phi}}{\partial t} d t d z-\int_{\mathbb{R}^{N}}\left(i \omega_{n} v^{0}+v^{1}\right) \bar{\phi}(0) d z .
$$

We further decompose the test function $\Phi_{\varepsilon}$ as follows

$$
\Phi_{\varepsilon}=\Phi_{\varepsilon}^{1}+\Phi_{\varepsilon}^{2} \cdot z \text { with } \Phi_{\varepsilon}^{2}=\sqrt{\varepsilon} \sum_{k=1}^{N} \frac{\partial \psi_{n}^{\varepsilon}}{\partial x_{k}} \phi(t, z) e_{k} .
$$

In the remaining terms of the variational formulation of (34), we replace $A^{\varepsilon}$ by its Taylor expansion (according to assumption H2) and we first look to those terms which are of zero order with respect to $z$. They are

$$
\begin{aligned}
& \int_{0}^{+\infty} \int_{\mathbb{R}^{N}}\left[A_{0}^{\varepsilon}\left(\nabla+\frac{2 \pi i \xi^{n}}{\sqrt{\varepsilon}}\right) v_{\varepsilon}^{+} \cdot\left(\left(\nabla-\frac{2 \pi i \xi^{n}}{\sqrt{\varepsilon}}\right) \overline{\Phi_{\varepsilon}^{1}}+\overline{\Phi_{\varepsilon}^{2}}\right)-\frac{1}{\varepsilon} \rho_{0}^{\varepsilon} \lambda_{n} \Phi_{\varepsilon}^{1}\right] d z d t \\
& =\int_{0}^{+\infty} \int_{\mathbb{R}^{N}}\left[\frac{1}{\varepsilon} A_{0}^{\varepsilon}\left(\sqrt{\varepsilon} \nabla+2 \pi i \xi^{n}\right) v_{\varepsilon}^{+} \cdot\left(\nabla_{y}-2 \pi i \xi^{n}\right) \bar{\psi}_{n}^{\varepsilon} \bar{\phi}-\frac{1}{\varepsilon} \rho_{0}^{\varepsilon} \lambda_{n} \bar{\psi}_{n}^{\varepsilon} v_{\varepsilon}^{+} \bar{\phi}\right] d z d t \\
& -\frac{1}{2 i \pi} \int_{0}^{+\infty} \int_{\mathbb{R}^{N}}\left[\frac{1}{\sqrt{\varepsilon}} A_{0}^{\varepsilon}\left(\sqrt{\varepsilon} \nabla+2 \pi i \xi^{n}\right) v_{\varepsilon}^{+} \cdot\left(\nabla_{y}-2 \pi i \xi^{n}\right) \frac{\partial \bar{\psi}_{n}^{\varepsilon}}{\partial \xi_{k}} \frac{\partial \bar{\phi}}{\partial z_{k}}\right. \\
& \left.-\frac{1}{\sqrt{\varepsilon}} \rho_{0}^{\varepsilon} \lambda_{n} v_{\varepsilon}^{+} \frac{\partial \bar{\psi}_{n}^{\varepsilon}}{\partial \xi_{k}} \frac{\partial \bar{\phi}}{\partial z_{k}}\right] d z d t \\
& +\int_{0}^{+\infty} \int_{\mathbb{R}^{N}} \frac{1}{\sqrt{\varepsilon}} A_{0}^{\varepsilon}\left(\sqrt{\varepsilon} \nabla+2 \pi i \xi^{n}\right) v_{\varepsilon}^{+} \cdot \bar{\psi}_{n}^{\varepsilon} \nabla \bar{\phi} d z d t \\
& +\int_{0}^{+\infty} \int_{\mathbb{R}^{N}}-\frac{1}{2 \pi i} A_{0}^{\varepsilon}\left(\sqrt{\varepsilon} \nabla+2 \pi i \xi^{n}\right) v_{\varepsilon}^{+} \cdot \frac{\partial \bar{\psi}_{n}^{\varepsilon}}{\partial \xi_{k}} \nabla \frac{\partial \bar{\phi}}{\partial z_{k}} d z d t \\
& +\int_{0}^{+\infty} \int_{\mathbb{R}^{N}} A_{0}^{\varepsilon}\left(\sqrt{\varepsilon} \nabla+2 \pi i \xi^{n}\right) v_{\varepsilon}^{+} \cdot \frac{\partial \bar{\psi}_{n}^{\varepsilon}}{\partial x_{k}} \bar{\phi} e_{k} d z d t .
\end{aligned}
$$


Using equation (20) with $\varphi=v_{\varepsilon}^{+} \bar{\phi}$ and equation (21) with $\varphi=v_{\varepsilon}^{+} \frac{\partial \bar{\phi}}{\partial z_{k}}$ we rewrite the first two integrals in the right hand side of (46) as follows

$$
\begin{aligned}
& \int_{0}^{+\infty} \int_{\mathbb{R}^{N}}-\frac{1}{\sqrt{\varepsilon}} A_{0}^{\varepsilon}\left(\nabla_{y}-2 \pi i \xi^{n}\right) \bar{\psi}_{n}^{\varepsilon} \cdot v_{\varepsilon}^{+} \nabla \bar{\phi} d z d t \\
& +\int_{0}^{+\infty} \int_{\mathbb{R}^{N}}\left[\frac{1}{2 \pi i} A_{0}^{\varepsilon}\left(\nabla_{y}-2 \pi i \xi^{n}\right) \frac{\partial \bar{\psi}_{n}^{\varepsilon}}{\partial \xi_{k}} \cdot v_{\varepsilon}^{+} \nabla \frac{\partial \bar{\phi}}{\partial z_{k}}+\frac{1}{\sqrt{\varepsilon}} A_{0}^{\varepsilon} e_{k} \cdot v_{\varepsilon}^{+} \frac{\partial \bar{\phi}}{\partial z_{k}}\left(\nabla_{y}-2 \pi i \xi^{n}\right) \bar{\psi}_{n}^{\varepsilon}\right. \\
& \left.\quad-\frac{1}{\sqrt{\varepsilon}} A_{0}^{\varepsilon} \bar{\psi}_{n}^{\varepsilon} e_{k} \cdot\left(\sqrt{\varepsilon} \nabla+2 \pi i \xi^{n}\right)\left(v_{\varepsilon}^{+} \frac{\partial \bar{\phi}}{\partial z_{k}}\right)\right] d z d t
\end{aligned}
$$

Combining the above terms with the other terms in (46) and passing to the two-scale limit in (46) yields

$$
\begin{aligned}
& \int_{0}^{+\infty} \int_{\mathbb{R}^{N}} \int_{\mathbb{T}^{N}}\left[\frac{1}{2 \pi i} A_{0} \psi_{n}\left(\nabla_{y}-2 \pi i \xi^{n}\right) \frac{\partial \bar{\psi}_{n}}{\partial \xi_{k}}-\frac{1}{2 \pi i} A_{0} \frac{\partial \bar{\psi}_{n}}{\partial \xi_{k}}\left(\nabla_{y}+2 \pi i \xi^{n}\right) \psi_{n}-A_{0}\left|\psi_{n}\right|^{2} e_{k}\right] \\
& \cdot v^{+} \nabla \frac{\partial \bar{\phi}}{\partial z_{k}} d y d z d t \\
& +\int_{0}^{+\infty} \int_{\mathbb{R}^{N}} \int_{\mathbb{T}^{N}} A_{0}\left(\nabla_{y}+2 i \pi \xi^{n}\right) \psi_{n} \cdot \frac{\partial \bar{\psi}_{n}}{\partial x_{k}} v^{+} \bar{\phi} e_{k} d y d z d t .
\end{aligned}
$$

By equation (19) the first integral of (47) is equal to

$$
\int_{0}^{+\infty} \int_{\mathbb{R}^{N}} A^{*} \nabla v^{+} \nabla \bar{\phi} d z d t,
$$

while the second integral of (47) contributes to the first term in formula (25) for $c^{*}$.

Next, we consider the terms which are linear in $z$ in the variational formulation of (34)

$$
\begin{aligned}
& \int_{0}^{+\infty} \int_{\mathbb{R}^{N}}\left[A_{0}^{\varepsilon}\left(\nabla+\frac{2 \pi i \xi^{n}}{\sqrt{\varepsilon}}\right) v_{\varepsilon}^{+} \cdot\left(z \nabla \bar{\Psi}_{\varepsilon}^{2}\right)-\frac{1}{\varepsilon} \rho_{0}^{\varepsilon} \lambda_{n} v_{\varepsilon}^{+} \bar{\Psi}_{\varepsilon}^{2} z\right] d z d t \\
& +\int_{0}^{+\infty} \int_{\mathbb{R}^{N}}\left[A_{1, k}^{\varepsilon} \sqrt{\varepsilon} z_{k}\left(\nabla+\frac{2 \pi i \xi^{n}}{\sqrt{\varepsilon}}\right) v_{\varepsilon}^{+} \cdot\left(\left(\nabla-\frac{2 \pi i \xi^{n}}{\sqrt{\varepsilon}}\right) \overline{\Psi_{\varepsilon}^{1}}+\overline{\Psi_{\varepsilon}^{2}}\right)\right] d z d t= \\
& \int_{0}^{+\infty} \int_{\mathbb{R}^{N}}\left[\frac{1}{\sqrt{\varepsilon}} A_{0}^{\varepsilon}\left(\sqrt{\varepsilon} \nabla+2 \pi i \xi^{n}\right) v_{\varepsilon}^{+} \cdot\left(\nabla_{y}-2 \pi i \xi^{n}\right) \frac{\partial \bar{\psi}_{n}^{\varepsilon}}{\partial x_{k}} \bar{\phi} z_{k}-\frac{1}{\sqrt{\varepsilon}} \lambda_{n} \rho_{0}^{\varepsilon} v_{\varepsilon}^{+} \frac{\partial \bar{\psi}_{n}^{\varepsilon}}{\partial x_{k}} z_{k}\right] d z d t \\
& \left.+\int_{0}^{+\infty} \int_{\mathbb{R}^{N}}^{\left[\frac{1}{\sqrt{\varepsilon}} A_{1, k}^{\varepsilon}\left(\sqrt{\varepsilon} \nabla+2 \pi i \xi^{n}\right) v_{\varepsilon}^{+} \cdot\left(\nabla_{y}-2 \pi i \xi^{n}\right) \bar{\psi}_{n}^{\varepsilon} \bar{\phi} z_{k}-\frac{1}{\sqrt{\varepsilon}} \lambda_{n} \rho_{1, k}^{\varepsilon} v_{\varepsilon}^{+} \bar{\psi}\right.} \bar{\phi} z_{k}\right] d z d t \\
& +\int_{0}^{+\infty} \int_{\mathbb{R}^{N}}\left[A_{0}^{\varepsilon}\left(\sqrt{\varepsilon} \nabla+2 \pi i \xi^{n}\right) v_{\varepsilon}^{+} \cdot \frac{\partial \bar{\psi}_{n}^{\varepsilon}}{\partial x_{k}} \nabla \bar{\phi} z_{k}+A_{1, k}^{\varepsilon}\left(\sqrt{\varepsilon} \nabla+2 \pi i \xi^{n}\right) v_{\varepsilon}^{+} \cdot \psi_{n}^{\varepsilon} \nabla \bar{\phi} z_{k}\right] d z d t \\
& -\frac{1}{2 \pi i} \int_{0}^{+\infty} \int_{\mathbb{R}^{N}}\left[A_{1, h}^{\varepsilon}\left(\sqrt{\varepsilon} \nabla+2 \pi i \xi^{n}\right) v_{\varepsilon}^{+} \cdot\left(\nabla_{y}-2 \pi i \xi^{n}\right) \frac{\partial \bar{\psi}_{n}^{\varepsilon}}{\partial \xi_{k}} \frac{\partial \bar{\phi}}{\partial z_{k}} z_{h}-\lambda_{n} \rho_{1, h}^{\varepsilon} v_{\varepsilon}^{+} \frac{\partial \bar{\psi}}{\partial \xi_{k}} \frac{\partial \bar{\phi}}{\partial z_{k}} z_{h}\right] d z d t \\
& +\int_{0}^{+\infty} \int_{\mathbb{R}^{N}}\left[\sqrt{\varepsilon} A_{1, h}^{\varepsilon}\left(\sqrt{\varepsilon} \nabla+2 \pi i \xi^{n}\right) v_{\varepsilon}^{+} \cdot\left(-\frac{1}{2 \pi i} \frac{\partial \bar{\psi}_{n}^{\varepsilon}}{\partial \xi_{k}} \nabla \frac{\partial \bar{\phi}}{\partial z_{k}}+\frac{\partial \bar{\psi}_{n}^{\varepsilon}}{\partial x_{k}} \bar{\phi} e_{k}\right) z_{h}\right] d z d t .
\end{aligned}
$$


By equation (22) with $\varphi=v_{\varepsilon}^{+} \bar{\phi} z_{k}$, and since $\nabla\left(\phi z_{k}\right)=\phi e_{k}+z_{k} \nabla \phi$, the sum of the first two integrals in the right hand side of (49) gives

$$
-\int_{0}^{+\infty} \int_{\mathbb{R}^{N}}\left(A_{0}^{\varepsilon}\left(\nabla_{y}-2 \pi i \xi^{n}\right) \frac{\partial \bar{\psi}_{n}^{\varepsilon}}{\partial x_{k}} \cdot v_{\varepsilon}^{+} \nabla\left(\bar{\phi} z_{k}\right)+A_{1, k}^{\varepsilon}\left(\nabla_{y}-2 \pi i \xi^{n}\right) \bar{\psi}_{n}^{\varepsilon} \cdot v_{\varepsilon}^{+} \nabla\left(\bar{\phi} z_{k}\right)\right) d z d t
$$

Therefore passing to the two-scale limit in (49) we have

$$
\begin{gathered}
-\int_{0}^{+\infty} \int_{\mathbb{R}^{N}} \int_{\mathbb{T}^{N}}\left[A_{0}\left(\nabla_{y}-2 \pi i \xi^{n}\right) \frac{\partial \bar{\psi}_{n}}{\partial x_{k}} \cdot v^{+} \psi_{n} \bar{\phi} e_{k}+A_{1, k}\left(\nabla_{y}-2 \pi i \xi^{n}\right) \bar{\psi}_{n} \cdot v^{+} \psi_{n} \bar{\phi} e_{k}\right] d y d z d t \\
-\int_{0}^{+\infty} \int_{\mathbb{R}^{N}} \int_{\mathbb{T}^{N}}\left[A_{0}\left(\nabla_{y}-2 \pi i \xi^{n}\right) \frac{\partial \bar{\psi}_{n}}{\partial x_{k}} \cdot v^{+} \psi_{n} z_{k} \nabla \bar{\phi}+A_{1, k}\left(\nabla_{y}-2 \pi i \xi^{n}\right) \bar{\psi}_{n} \cdot v^{+} \psi_{n} z_{k} \nabla \bar{\phi}\right] d y d z d t \\
+\int_{0}^{+\infty} \int_{\mathbb{R}^{N}} \int_{\mathbb{T}^{N}}\left[A_{0}\left(\nabla_{y}+2 \pi i \xi^{n}\right) \psi_{n} \cdot v^{+} \frac{\partial \bar{\psi}_{n}}{\partial x_{k}} z_{k} \nabla \bar{\phi}+A_{1, k}\left(\nabla_{y}+2 \pi i \xi^{n}\right) \psi_{n} \cdot v^{+} \bar{\psi}_{n} z_{k} \nabla \bar{\phi}\right] d y d z d t \\
-\frac{1}{2 \pi i} \int_{0}^{+\infty} \int_{\mathbb{R}^{N}} \int_{\mathbb{T}^{N}}\left[A_{1, h}\left(\nabla_{y}+2 \pi i \xi^{n}\right) \psi_{n} \cdot\left(\nabla_{y}-2 \pi i \xi^{n}\right) \frac{\partial \bar{\psi}_{n}}{\partial \xi_{k}} v^{+} z_{h} \frac{\partial \bar{\phi}}{\partial z_{k}}\right. \\
\left.-\lambda_{n} \rho_{1, h} v^{+} \psi_{n} \frac{\partial \bar{\psi}_{n}}{\partial \xi_{k}} \frac{\partial \bar{\phi}_{k}}{\partial z_{k}} z_{h}\right] d y d z d t .
\end{gathered}
$$

By equation (17) it follows that the last integral in (51) is equal to

$$
\begin{aligned}
& \int_{0}^{+\infty} \int_{\mathbb{R}^{N}} \int_{\mathbb{T}^{N}}\left[A_{1, h} \psi_{n} e_{k} \cdot\left(\nabla_{y}-2 \pi i \xi^{n}\right) \bar{\psi}_{n}+A_{0} \psi_{n} e_{k} \cdot\left(\nabla_{y}-2 \pi i \xi^{n}\right) \frac{\partial \bar{\psi}_{n}}{\partial x_{h}}\right] v^{+} z_{h} \frac{\partial \bar{\phi}}{\partial z_{k}} d y d z d t \\
& -\int_{0}^{+\infty} \int_{\mathbb{R}^{N}} \int_{\mathbb{T}^{N}}\left[A_{1, h} \bar{\psi}_{n} e_{k} \cdot\left(\nabla_{y}+2 \pi i \xi^{n}\right) \psi_{n}+A_{0} \frac{\partial \bar{\psi}_{n}}{\partial x_{h}} e_{k} \cdot\left(\nabla_{y}+2 \pi i \xi^{n}\right) \psi_{n}\right] v^{+} z_{h} \frac{\partial \bar{\phi}}{\partial z_{k}} d y d z d t \\
& -\int_{0}^{+\infty} \int_{\mathbb{R}^{N}} \int_{\mathbb{T}^{N}} \frac{1}{2 \pi i} \frac{\partial^{2} \lambda_{n}}{\partial x_{h} \partial \xi_{k}}\left|\psi_{n}\right|^{2} v^{+} z_{h} \frac{\partial \bar{\phi}}{\partial z_{k}} d y d z d t .
\end{aligned}
$$

Notice that the first and the second line of (52) cancel out with the second and third line of (51) respectively and therefore (51) reduces to

$$
\begin{aligned}
& -\int_{0}^{+\infty} \int_{\mathbb{R}^{N}} \int_{\mathbb{T}^{N}}\left[A_{0}\left(\nabla_{y}-2 \pi i \xi^{n}\right) \frac{\partial \bar{\psi}_{n}}{\partial x_{k}} \cdot v^{+} \psi_{n} \bar{\phi} e_{k}+A_{1, k}\left(\nabla_{y}-2 \pi i \xi^{n}\right) \bar{\psi}_{n} \cdot v^{+} \psi_{n} \bar{\phi} e_{k}\right] d y d z d t \\
& -\int_{0}^{+\infty} \int_{\mathbb{R}^{N}} \frac{1}{2 i \pi} \frac{\partial^{2} \lambda_{n}}{\partial x_{h} \partial \xi_{k}} v^{+} \frac{\partial \bar{\phi}}{\partial z_{k}} z_{h} d z d t .
\end{aligned}
$$


Finally we consider all quadratic in $z$ terms in the variational formulation of (34)

$$
\begin{aligned}
& \frac{1}{2} \int_{0}^{+\infty} \int_{\mathbb{R}^{N}}\left[A_{2, l h}^{\varepsilon} \varepsilon z_{l} z_{h}\left(\nabla+\frac{2 i \pi \xi^{n}}{\sqrt{\varepsilon}}\right) v_{\varepsilon}^{+} \cdot\left(\left(\nabla-\frac{2 i \pi \xi^{n}}{\sqrt{\varepsilon}}\right) \bar{\Psi}_{\varepsilon}^{1}+\bar{\Psi}_{\varepsilon}^{2}\right)-\lambda_{n} \rho_{2, l h}^{\varepsilon} z_{l} z_{h} v_{\varepsilon}^{+} \bar{\Psi}_{\varepsilon}^{1}\right] d z d t \\
& +\int_{0}^{+\infty} \int_{\mathbb{R}^{N}}\left[A_{1, k}^{\varepsilon} \varepsilon z_{k}\left(\nabla-\frac{2 i \pi \xi^{n}}{\sqrt{\varepsilon}}\right) v_{\varepsilon}^{+} \cdot\left(z \nabla \bar{\Psi}_{\varepsilon}^{2}\right)-\lambda_{n} \rho_{1, k}^{\varepsilon} z_{k} v_{\varepsilon}^{+} z \cdot \bar{\Psi}_{\varepsilon}^{2}\right] d z d t \\
& =\frac{1}{2} \int_{0}^{+\infty} \int_{\mathbb{R}^{N}} A_{2, l h}^{\varepsilon} \varepsilon z_{l} z_{h}\left(\sqrt{\varepsilon} \nabla+2 \pi i \xi^{n}\right) v_{\varepsilon}^{+} \cdot\left(\nabla_{y}-2 \pi i \xi^{n}\right) \bar{\psi}_{n}^{\varepsilon} \bar{\phi} d z d t \\
& +\frac{1}{2} \int_{0}^{+\infty} \int_{\mathbb{R}^{N}} A_{1, h}^{\varepsilon} z_{h}\left(\sqrt{\varepsilon} \nabla+2 \pi i \xi^{n}\right) v_{\varepsilon}^{+} \cdot z_{k}\left(\nabla_{y}-2 \pi i \xi^{n}\right) \frac{\partial \bar{\psi}_{n}^{\varepsilon}}{\partial x_{k}} \bar{\phi} d z d t \\
& -\int_{0}^{+\infty} \int_{\mathbb{R}^{N}} \frac{1}{2} \lambda_{n} \rho_{2, l h}^{\varepsilon} z_{l} z_{h} v_{\varepsilon}^{+} \bar{\psi}_{n}^{\varepsilon} \bar{\phi} d z d t-\int_{0}^{+\infty} \int_{\mathbb{R}^{N}} \lambda_{n} \rho_{1, h}^{\varepsilon} z_{h} v_{\varepsilon}^{+} z_{k} \frac{\partial \bar{\psi}_{n}^{\varepsilon}}{\partial x_{k}} \bar{\phi} d z d t+o(1)
\end{aligned}
$$

which give on passing to the two-scale limit

$$
\begin{aligned}
& \frac{1}{2} \int_{0}^{+\infty} \int_{\mathbb{R}^{N}} \int_{\mathbb{T}^{N}}\left[A_{2, l h}\left(\nabla_{y}+2 \pi i \xi^{n}\right) \psi_{n} \cdot\left(\nabla_{y}-2 \pi i \xi^{n}\right) \bar{\psi}_{n}-\lambda_{n} \rho_{2, l h} \psi_{n} \bar{\psi}_{n}\right] v^{+} \bar{\phi} z_{l} z_{h} d y d z d t \\
& +\int_{0}^{+\infty} \int_{\mathbb{R}^{N}} \int_{\mathbb{T}^{N}}\left[A_{1, h}\left(\nabla_{y}+2 \pi i \xi^{n}\right) \psi_{n} \cdot\left(\nabla_{y}-2 \pi i \xi^{n}\right) \frac{\partial \bar{\psi}_{n}}{\partial x_{k}}-\lambda_{n} \rho_{1, h} \psi_{n} \frac{\partial \bar{\psi}_{n}}{\partial x_{k}}\right] v^{+} \bar{\phi} z_{l} z_{h} d y d z d t
\end{aligned}
$$

Now using the equation (18) we find that (55) reduces to

$$
\int_{0}^{+\infty} \int_{\mathbb{R}^{N}} \frac{1}{2} \frac{\partial^{2} \lambda_{n}}{\partial x_{l} \partial x_{h}} v^{+} \bar{\phi} z_{l} z_{h} d z d t
$$

Summing up together (45), (47), (48), (53) and (56) yields the weak formulation of (24). We know by Proposition 3.5 that (24) admits a unique solution. Therefore, the entire sequence $v_{\varepsilon}^{+}$, and not merely a subsequence, converges. Of course a symmetric proof works for the other sequence $v_{\varepsilon}^{-}$corresponding to the opposite phase.

\section{Strong convergence (proof of Theorem 3.2)}

Recall that $u_{\varepsilon}^{\text {approx }}$ has a $L^{2}$-norm of order $\varepsilon^{N / 4}$, so we expect the same for $u_{\varepsilon}$. Therefore we define the difference between them in the scale of the $z$-variable. In other words we define $r_{\varepsilon}(t, z)$ by

$$
r_{\varepsilon}\left(t, \frac{x}{\sqrt{\varepsilon}}\right)=u_{\varepsilon}(t, x)-\psi_{n}\left(\frac{x}{\varepsilon}\right) e^{2 i \pi \frac{\xi^{n} \cdot x}{\varepsilon}}\left(e^{\frac{i \omega_{n} t}{\epsilon}} v^{+}\left(t, \frac{x}{\sqrt{\varepsilon}}\right)+e^{\frac{-i \omega_{n} t}{\epsilon}} v^{-}\left(t, \frac{x}{\sqrt{\varepsilon}}\right)\right) .
$$

Theorem 3.2 amounts to prove that $r_{\varepsilon}(t, z)$ converges strongly to zero in $L^{2}\left((0, T) \times \mathbb{R}^{N}\right)$.

We begin with a technical lemma, the proof of which is postponed for a moment.

Lemma 6.1 The solution of (1) satisfies

$$
\lim _{\varepsilon \rightarrow 0} \frac{1}{\varepsilon^{N / 2}} \int_{0}^{T} \int_{\mathbb{R}^{N}} \rho\left(x, \frac{x}{\varepsilon}\right)\left|u_{\varepsilon}(t, x)\right|^{2} d x d t=\left\|v^{+}\right\|_{L^{2}\left((0, T) \times \mathbb{R}^{N}\right)}^{2}+\left\|v^{-}\right\|_{L^{2}\left((0, T) \times \mathbb{R}^{N}\right)}^{2}
$$


Equipped with Lemma 6.1 we are now able to prove Theorem 3.2. We develop the following norm

$$
\begin{array}{r}
\int_{0}^{T} \int_{\mathbb{R}^{N}} \rho\left(\sqrt{\varepsilon} z, \frac{z}{\sqrt{\varepsilon}}\right)\left|r_{\varepsilon}(t, z)\right|^{2} d z d t=\frac{1}{\varepsilon^{N / 2}} \int_{0}^{T} \int_{\mathbb{R}^{N}} \rho\left(x, \frac{x}{\varepsilon}\right)\left|r_{\varepsilon}\left(t, \frac{x}{\sqrt{\varepsilon}}\right)\right|^{2} d x d t \\
=\frac{1}{\varepsilon^{N / 2}} \int_{0}^{T} \int_{\mathbb{R}^{N}} \rho\left(x, \frac{x}{\varepsilon}\right)\left|u_{\varepsilon}(t, x)\right|^{2} d x d t \\
+\frac{1}{\varepsilon^{N / 2}} \int_{0}^{T} \int_{\mathbb{R}^{N}} \rho\left(x, \frac{x}{\varepsilon}\right)\left|\psi_{n}\left(\frac{x}{\varepsilon}\right) v^{+}\left(t, \frac{x}{\sqrt{\varepsilon}}\right)\right|^{2} d x d t \\
+\frac{1}{\varepsilon^{N / 2}} \int_{0}^{T} \int_{\mathbb{R}^{N}} \rho\left(x, \frac{x}{\varepsilon}\right)\left|\psi_{n}\left(\frac{x}{\varepsilon}\right) v^{-}\left(t, \frac{x}{\sqrt{\varepsilon}}\right)\right|^{2} d x d t \\
-\frac{2}{\varepsilon^{N / 2}} \mathcal{R} \int_{0}^{T} \int_{\mathbb{R}^{N}} \rho\left(x, \frac{x}{\varepsilon}\right) v_{\varepsilon}^{+}(t, x) \bar{\psi}_{n}\left(\frac{x}{\varepsilon}\right) \overline{v^{+}}\left(t, \frac{x}{\sqrt{\varepsilon}}\right) d x d t \\
-\frac{2}{\varepsilon^{N / 2}} \mathcal{R} \int_{0}^{T} \int_{\mathbb{R}^{N}} \rho\left(x, \frac{x}{\varepsilon}\right) v_{\varepsilon}^{-}(t, x) \bar{\psi}_{n}\left(\frac{x}{\varepsilon}\right) \frac{x}{v^{-}}\left(t, \frac{x}{\sqrt{\varepsilon}}\right) d x d t \\
+\frac{2}{\varepsilon^{N / 2}} \mathcal{R} \int_{0}^{T} \int_{\mathbb{R}^{N}} \rho\left(x, \frac{x}{\varepsilon}\right)\left|\psi_{n}\left(\frac{x}{\varepsilon}\right)\right|^{2} \frac{x}{v^{+}}\left(t, \frac{x}{\sqrt{\varepsilon}}\right) v^{-}\left(t, \frac{x}{\sqrt{\varepsilon}}\right) e^{\frac{-2 i \omega_{n} t}{\varepsilon}} d x d t
\end{array}
$$

where $v_{\varepsilon}^{+}, v_{\varepsilon}^{-}$are defined by (23) and (26), while $\mathcal{R}$ denotes the real part of a complex number. By virtue of Lemma 6.1 we can pass to the limit in the first integral in the right hand side of (59). By using the changes of variables $z=\frac{x}{\sqrt{\varepsilon}}$, and recalling that

$$
\left|\rho\left(\sqrt{\varepsilon} z, \frac{z}{\sqrt{\varepsilon}}\right)-\rho\left(0, \frac{z}{\sqrt{\varepsilon}}\right)\right| \leq C \min \{\sqrt{\varepsilon} z, 1\},
$$

it is easy to pass to the limit in the second and third integrals in the right hand side of (59) to obtain

$$
\lim _{\varepsilon \rightarrow 0} \frac{1}{\varepsilon^{N / 2}} \int_{0}^{T} \int_{\mathbb{R}^{N}} \rho\left(x, \frac{x}{\varepsilon}\right)\left|\psi_{n}\left(\frac{x}{\varepsilon}\right) v^{ \pm}\left(t, \frac{x}{\sqrt{\varepsilon}}\right)\right|^{2} d x d t=\left\|v^{ \pm}\right\|_{L^{2}\left((0, T) \times \mathbb{R}^{N}\right)}^{2} .
$$

We pass to the two-scale limit in the fourth and fifth integrals in the right hand side of (59)

$$
\begin{array}{r}
\lim _{\varepsilon \rightarrow 0} \frac{2}{\varepsilon^{N / 2}} \mathcal{R} \int_{0}^{T} \int_{\mathbb{R}^{N}} \rho\left(x, \frac{x}{\varepsilon}\right) v_{\varepsilon}^{ \pm}(t, x) \bar{\psi}_{n}\left(\frac{x}{\varepsilon}\right) \overline{v^{ \pm}}\left(t, \frac{x}{\sqrt{\varepsilon}}\right) d x d t \\
=2 \mathcal{R} \int_{0}^{T} \int_{\mathbb{R}^{N}} \int_{\mathbb{T}^{N}} \rho_{0}(y)\left|\psi_{n}(y)\right|^{2}\left|v^{ \pm}(t, z)\right|^{2} d y d z d t=2\left\|v^{ \pm}\right\|_{L^{2}\left((0, T) \times \mathbb{R}^{N}\right)}^{2} .
\end{array}
$$

To show that the limit of the last integral in the right hand side of (59) is actually zero we take advantage of the time oscillating phase. We define

$$
K_{\varepsilon}(t)=\frac{1}{\varepsilon^{N / 2}} \int_{\mathbb{R}^{N}} \rho\left(x, \frac{x}{\varepsilon}\right)\left|\psi_{n}\left(\frac{x}{\varepsilon}\right)\right|^{2} v^{+}\left(t, \frac{x}{\sqrt{\varepsilon}}\right) v^{-}\left(t, \frac{x}{\sqrt{\varepsilon}}\right) d x
$$


which is a continuous function of time (because $v^{ \pm}$belong to $C\left(\mathbb{R}^{+} ; L^{2}\left(\mathbb{R}^{N}\right)\right)$ ) and converges uniformly to a continuous limit $K(t)$ as $\varepsilon$ goes to 0 . Therefore, since $\omega_{n} \neq 0$,

$$
\lim _{\varepsilon \rightarrow 0} \int_{0}^{T} K_{\varepsilon}(t) e^{\frac{-2 i \omega_{n} t}{\varepsilon}} d t=0 .
$$

We thus obtain

$$
\lim _{\varepsilon \rightarrow 0} \int_{0}^{T} \int_{\mathbb{R}^{N}} \rho\left(\sqrt{\varepsilon} z, \frac{z}{\sqrt{\varepsilon}}\right)\left|r_{\varepsilon}(t, z)\right|^{2} d z d t=2\left\|v^{+}\right\|^{2}+2\left\|v^{-}\right\|^{2}-2\left\|v^{+}\right\|^{2}-2\left\|v^{-}\right\|^{2}=0
$$

which completes the proof of Theorem 3.2.

It remains to prove Lemma 6.1, a long task partitioned in several other lemmas. The main idea is to use the energy conservations for the $\varepsilon$-oscillating wave equation and the homogenized Schrödinger equations and show that the initial energy of the wave equation converges to the sum of the initial energies of the two homogenized Schrödinger equations. There are additional technical difficulties. First, the left hand side of (58) does not involve the energy of the wave equation (1): therefore we rely on the energy of a time primitive of (1) (as introduced in Section 4) and on an energy equipartition (see Lemma 6.5). Second, refined a priori estimates are necessary and a second time regularization has to be introduced (see Lemmas 6.4 and 6.2).

Let us start by recalling the definition (36) of the time primitive of the solution $u_{\varepsilon}$ of (1)

$$
\Psi_{\varepsilon}(x, t)=e^{-\alpha t}\left[\int_{0}^{t} e^{\alpha s} u_{\varepsilon}(x, s) d s+\chi_{\varepsilon}(x)\right]
$$

with $\chi_{\varepsilon}$ solution of (37). We know that $\Psi_{\varepsilon}$ is the solution of another wave equation, (38). We improve the a priori estimate of Lemma 4.1 on the initial data $\chi_{\varepsilon}(x)$. Surprisingly the result is different according to the value of $\xi^{n}$.

Lemma 6.2 Define the sequence

$$
w_{\varepsilon}(z)=\frac{1}{\varepsilon} \chi_{\varepsilon}(\sqrt{\varepsilon} z) e^{-2 i \pi \frac{\xi^{n} \cdot z}{\sqrt{\varepsilon}}}
$$

If $\xi^{n} \neq 0$, it satisfies

$$
\sqrt{\varepsilon}\left\|\nabla_{z} w_{\varepsilon}\right\|_{L^{2}\left(\mathbb{R}^{N}\right)}+\left\|w_{\varepsilon}\right\|_{L^{2}\left(\mathbb{R}^{N}\right)} \leq C\left(\xi^{n}\right)\left(\left\|v^{0}\right\|_{L^{2}\left(\mathbb{R}^{N}\right)}+\left\|v^{1}\right\|_{H^{2}\left(\mathbb{R}^{N}\right)}\right),
$$

where the constant $C\left(\xi^{n}\right)$ does not depend on $\varepsilon$, and it two-scale converges strongly to $-\frac{\psi_{n}(y)}{\lambda_{n}} v^{1}(z)$, while $\sqrt{\varepsilon} \nabla w_{\varepsilon}(z)$ two-scale converges strongly to $-\frac{\nabla_{y} \psi_{n}(y)}{\lambda_{n}} v^{1}(z)$.

If $\xi^{n}=0$, it satisfies

$$
\sqrt{\varepsilon}\left\|\nabla_{z} w_{\varepsilon}\right\|_{L^{2}\left(\mathbb{R}^{N}\right)}+\sqrt{\varepsilon}\left\|w_{\varepsilon}\right\|_{L^{2}\left(\mathbb{R}^{N}\right)} \leq C\left(\left\|v^{0}\right\|_{L^{2}\left(\mathbb{R}^{N}\right)}+\left\|v^{1}\right\|_{H^{2}\left(\mathbb{R}^{N}\right)}\right),
$$

where the constant $C\left(\xi^{n}\right)$ does not depend on $\varepsilon$, and $\sqrt{\varepsilon} \nabla w_{\varepsilon}(z)$ two-scale converges strongly to $-\frac{\nabla_{y} \psi_{n}(y)}{\lambda_{n}} v^{1}(z)$ 

for $\Psi_{\varepsilon}$

We postpone for a moment the proof of Lemma 6.2 and we introduce the energy functional

$$
E\left(\Psi_{\varepsilon}\right)(t)=\frac{1}{2} \int_{\mathbb{R}^{N}}\left(\rho\left(x, \frac{x}{\varepsilon}\right)\left|\frac{\partial \Psi_{\varepsilon}}{\partial t}\right|^{2}+A\left(x, \frac{x}{\varepsilon}\right) \nabla \Psi_{\varepsilon} \cdot \nabla \bar{\Psi}_{\varepsilon}\right) d x .
$$

Lemma 6.3 The energy of the initial data satisfies

$$
\lim _{\varepsilon \rightarrow 0} \frac{1}{\varepsilon^{N / 2}} E\left(\Psi_{\varepsilon}\right)(0)=\frac{1}{2}\left(\left\|v^{0}\right\|_{L^{2}\left(\mathbb{R}^{N}\right)}^{2}+\frac{1}{\omega_{n}^{2}}\left\|v^{1}\right\|_{L^{2}\left(\mathbb{R}^{N}\right)}^{2}\right) .
$$

Proof. In view of the wave equation (38) we have

$$
E\left(\Psi_{\varepsilon}\right)(0)=\frac{1}{2} \int_{\mathbb{R}^{N}}\left(\rho\left(x, \frac{x}{\varepsilon}\right)\left|u_{\varepsilon}^{0}(x)-\alpha \chi_{\varepsilon}(x)\right|^{2}+A\left(x, \frac{x}{\varepsilon}\right) \nabla \chi_{\varepsilon}(x) \cdot \nabla \bar{\chi}_{\varepsilon}(x)\right) d x
$$

By the change of variables $z=\frac{x}{\sqrt{\varepsilon}}$ we rewrite

$$
E\left(\Psi_{\varepsilon}\right)(0)=\frac{\varepsilon^{N / 2}}{2} \int_{\mathbb{R}^{N}}\left(\tilde{\rho}^{\varepsilon}\left|\tilde{u}_{\varepsilon}^{0}(z)-\alpha \tilde{\chi}_{\varepsilon}(z)\right|^{2}+\frac{1}{\varepsilon} \tilde{A}^{\varepsilon} \nabla_{z} \tilde{\chi}_{\varepsilon}(z) \cdot \nabla_{z} \overline{\tilde{\chi}}_{\varepsilon}(z)\right) d z
$$

with the notation (33) for $\tilde{\rho}^{\varepsilon}(x)=\rho\left(\sqrt{\varepsilon} z, \frac{z}{\sqrt{\varepsilon}}\right)$ (similarly for other functions) which satisfies (60). From Lemma 6.2 we deduce that, as $\varepsilon$ goes to 0 ,

$$
\int_{\mathbb{R}^{N}} \tilde{\rho}^{\varepsilon}\left|\tilde{u}_{\varepsilon}^{0}-\alpha \tilde{\chi}_{\varepsilon}\right|^{2} d z=\int_{\mathbb{R}^{N}} \tilde{\rho}^{\varepsilon}\left|\tilde{\psi}_{n}^{\varepsilon} v^{0}-\alpha \varepsilon w_{\varepsilon}\right|^{2} d z \rightarrow\left\|v^{0}\right\|_{L^{2}\left(\mathbb{R}^{N}\right)}^{2}
$$

because, for any value of $\xi^{n}, \sqrt{\varepsilon} w_{\varepsilon}$ is bounded in $L^{2}\left(\mathbb{R}^{N}\right)$, and

$$
\int_{\mathbb{R}^{N}}\left(\frac{1}{\varepsilon} \tilde{A}^{\varepsilon} \nabla_{z} \tilde{\chi}_{\varepsilon} \cdot \nabla_{z} \overline{\tilde{\chi}}_{\varepsilon}\right) d z=\int_{\mathbb{R}^{N}} \tilde{A}^{\varepsilon}\left(\sqrt{\varepsilon} \nabla_{z}+2 i \pi \xi^{n}\right) w_{\varepsilon} \cdot\left(\sqrt{\varepsilon} \nabla_{z}-2 i \pi \xi^{n}\right) \bar{w}_{\varepsilon} d z
$$

converges to

$$
\frac{1}{\lambda_{n}^{2}} \int_{\mathbb{R}^{N}} \int_{\mathbb{T}^{N}} A_{0}(y)\left(\nabla_{y}+2 i \pi \xi^{n}\right) \psi_{n}(y) \cdot\left(\nabla_{y}-2 i \pi \xi^{n}\right) \bar{\psi}_{n}(y)\left|v^{1}(z)\right|^{2} d y d z=\frac{1}{\lambda_{n}} \int_{\mathbb{R}^{N}}\left|v^{1}(z)\right|^{2} d z
$$

because of the strong two-scale convergence of Lemma 6.2 (remark that, when $\xi^{n}=0$, we do not need the convergence of $w_{\varepsilon}$ ). Summing these two limits finishes the proof of Lemma 6.3 .

Although $\Psi_{\varepsilon}$ has a finite non-vanishing energy, as just shown in Lemma 6.3, its amplitude is asymptotically vanishing (but of course not its first derivatives). 
Lemma 6.4 For any final time $T>0$ there exists a constant $C(T)>0$, independent of $\varepsilon$, such that

$$
\left\|\Psi_{\varepsilon}\right\|_{L^{\infty}\left((0, T) ; L^{2}\left(\mathbb{R}^{N}\right)\right.} \leq C(T) \varepsilon^{N / 4+1 / 2}\left(\left\|v^{0}\right\|_{H^{2}\left(\mathbb{R}^{N}\right)}+\left\|v^{1}\right\|_{H^{2}\left(\mathbb{R}^{N}\right)}\right) .
$$

Proof. As we did in Section 4 where we introduced a time primitive $\Psi_{\varepsilon}$ of $u_{\varepsilon}$, we now iterate this regularization procedure and introduce a new time primitive $\Phi_{\varepsilon}$ of $\Psi_{\varepsilon}$, defined by

$$
\Phi_{\varepsilon}(x, t)=e^{-t}\left[\int_{0}^{t} e^{t} \Psi_{\varepsilon}(x, s) d s+\pi_{\varepsilon}\right]
$$

where $\pi_{\varepsilon}$ is the unique solution in $H^{1}\left(\mathbb{R}^{N}\right)$ of the equation

$$
-\operatorname{div}\left(A\left(x, \frac{x}{\varepsilon}\right) \nabla \pi_{\varepsilon}\right)+\rho\left(x, \frac{x}{\varepsilon}\right) \pi_{\varepsilon}=2 \rho\left(x, \frac{x}{\varepsilon}\right) \chi_{\varepsilon}-\rho\left(x, \frac{x}{\varepsilon}\right) u_{\varepsilon}^{0} .
$$

Then, $\Phi_{\varepsilon}$ satisfies

$$
\left\{\begin{aligned}
\rho\left(x, \frac{x}{\varepsilon}\right) \frac{\partial^{2} \Phi_{\varepsilon}}{\partial t^{2}}-\operatorname{div}\left(A\left(x, \frac{x}{\varepsilon}\right) \nabla \Phi_{\varepsilon}\right) & =0 & & \text { in } \mathbb{R}^{+} \times \mathbb{R}^{N} \\
\Phi_{\varepsilon}(0) & =\pi_{\varepsilon} & & \text { in } \mathbb{R}^{N} \\
\frac{\partial \Phi_{\varepsilon}}{\partial t}(0) & =\chi_{\varepsilon}-\pi_{\varepsilon} & & \text { in } \mathbb{R}^{N} .
\end{aligned}\right.
$$

From Lemma 6.2 we know that

$$
\left\|\chi_{\varepsilon}\right\|_{L^{2}\left(\mathbb{R}^{N}\right)} \leq C \varepsilon^{N / 4+1 / 2}\left(\left\|v^{0}\right\|_{L^{2}\left(\mathbb{R}^{N}\right)}+\left\|v^{1}\right\|_{H^{2}\left(\mathbb{R}^{N}\right)}\right) .
$$

Remark that the estimate (67) is optimal when $\xi^{n}=0$ but is pessimistic when $\xi^{n} \neq 0$ because the factor $\varepsilon^{N / 4+1 / 2}$ could be replaced by $\varepsilon^{N / 4+1}$. However, we do not need this refinement in the sequel. Reproducing the arguments of the proof of Lemma 4.1 we can prove that

$$
\left|\int_{\mathbb{R}^{N}} \rho\left(x, \frac{x}{\varepsilon}\right) u_{\varepsilon}^{0} \overline{\pi_{\varepsilon}}(x) d x\right| \leq C \varepsilon^{N / 4+1}\left\|v^{0}\right\|_{H^{2}\left(\mathbb{R}^{N}\right)}\left\|\pi_{\varepsilon}\right\|_{H^{1}\left(\mathbb{R}^{N}\right)} .
$$

Therefore, from the energy equality of (65) we deduce

$$
\left\|\pi_{\varepsilon}\right\|_{H^{1}\left(\mathbb{R}^{N}\right)} \leq C \varepsilon^{N / 4+1 / 2}\left(\left\|v^{0}\right\|_{H^{2}\left(\mathbb{R}^{N}\right)}+\left\|v^{1}\right\|_{H^{2}\left(\mathbb{R}^{N}\right)}\right) .
$$

The standard energy conservation for (66), together with (67) and (68), imply that

$$
\left\|\frac{\partial \Phi_{\varepsilon}}{\partial t}\right\| \leq C \varepsilon^{N / 4+1 / 2}\left(\left\|v^{0}\right\|_{H^{2}\left(\mathbb{R}^{N}\right)}+\left\|v^{1}\right\|_{H^{2}\left(\mathbb{R}^{N}\right)}\right)
$$

which, combined with the relations

$$
\Phi_{\varepsilon}(t)=\pi_{\varepsilon}+\int_{0}^{t} \frac{\partial \Phi_{\varepsilon}}{\partial t}(s) d s \quad \text { and } \quad \Psi_{\varepsilon}=\frac{\partial \Phi_{\varepsilon}}{\partial t}+\Phi_{\varepsilon},
$$

yields the desired result (64).

We now prove a result on the equipartition of the energy saying that the kinetic energy is essentially equal to the deformation energy. 
Lemma 6.5 The following equality holds

$$
\int_{0}^{T} E\left(\Psi_{\varepsilon}(t)\right) d t=\int_{0}^{T} \int_{\mathbb{R}^{N}} \rho\left(x, \frac{x}{\varepsilon}\right)\left|\frac{\partial \Psi_{\varepsilon}}{\partial t}\right|^{2} d x d t+o\left(\varepsilon^{N / 2}\right) .
$$

Proof. We multiply equation (38) by $\bar{\Psi}_{\varepsilon}(t, x) \phi(t)$, where $\phi$ is a smooth function defined on $\mathbb{R}^{+}$. Integrating by parts we obtain

$$
\begin{aligned}
& -\int_{0}^{T} \int_{\mathbb{R}^{N}} \phi(t) \rho\left(x, \frac{x}{\varepsilon}\right)\left|\frac{\partial \Psi_{\varepsilon}}{\partial t}\right|^{2} d x d t+\int_{0}^{T} \int_{\mathbb{R}^{N}} \phi(t) A\left(x, \frac{x}{\varepsilon}\right) \nabla \Psi_{\varepsilon} \cdot \nabla \bar{\Psi}_{\varepsilon} d x d t \\
& -\int_{0}^{T} \frac{\partial \phi}{\partial t}(t) \int_{\mathbb{R}^{N}} \rho\left(x, \frac{x}{\varepsilon}\right) \frac{\partial \Psi_{\varepsilon}}{\partial t} \bar{\Psi}_{\varepsilon} d x d t+\left[\int_{\mathbb{R}^{N}} \phi(t) \rho\left(x, \frac{x}{\varepsilon}\right) \frac{\partial \Psi_{\varepsilon}}{\partial t} \bar{\Psi}_{\varepsilon} d x\right]_{0}^{T}=0 .
\end{aligned}
$$

From (64) and (40) we have that, for a.e. $t \in[0, T]$,

$$
\left|\int_{\mathbb{R}^{N}} \rho\left(x, \frac{x}{\varepsilon}\right) \frac{\partial \Psi_{\varepsilon}}{\partial t} \bar{\Psi}_{\varepsilon} d x\right| \leq C(T) \varepsilon^{N / 2+1 / 2} .
$$

Thus, (70) can be rewritten as

$$
\int_{0}^{T} \phi(t)\left[\int_{\mathbb{R}^{N}} \rho\left(x, \frac{x}{\varepsilon}\right)\left|\frac{\partial \Psi_{\varepsilon}}{\partial t}\right|^{2} d x-\int_{\mathbb{R}^{N}} A\left(x, \frac{x}{\varepsilon}\right) \nabla \Psi_{\varepsilon} \cdot \nabla \bar{\Psi}_{\varepsilon} d x\right] d t=o\left(\varepsilon^{N / 2}\right) .
$$

Taking $\phi \equiv 1$ yields the desired result.

Proof of Lemma 6.1. The limit of the initial energy for the solution $\Psi_{\varepsilon}$ of the wave equation (38) is given by Lemma 6.3 and it coincides with the sum of the initial energies of the homogenized Schrödinger equations (24) and (27). Indeed, we have

$$
\left\|v^{+}(0, \cdot)\right\|_{L^{2}\left(\mathbb{R}^{N}\right)}^{2}+\left\|v^{-}(0, \cdot)\right\|_{L^{2}\left(\mathbb{R}^{N}\right)}^{2}=\frac{1}{2}\left(\left\|v^{0}\right\|_{L^{2}\left(\mathbb{R}^{N}\right)}^{2}+\frac{1}{\omega_{n}^{2}}\left\|v^{1}\right\|_{L^{2}\left(\mathbb{R}^{N}\right)}^{2}\right)=\lim _{\varepsilon \rightarrow 0} \frac{1}{\varepsilon^{N / 2}} E\left(\Psi_{\varepsilon}\right)(0) .
$$

From this equality, using the energy conservations for the wave equation (38) and the homogenized Schrödinger equations (as proved in Proposition 3.5), we deduce

$$
\left\|v^{+}(t, \cdot)\right\|_{L^{2}\left(\mathbb{R}^{N}\right)}^{2}+\left\|v^{-}(t, \cdot)\right\|_{L^{2}\left(\mathbb{R}^{N}\right)}^{2}=\lim _{\varepsilon \rightarrow 0} \frac{1}{\varepsilon^{N / 2}} E\left(\Psi_{\varepsilon}\right)(t) .
$$

Integrating in time and recalling the energy equipartition of Lemma 6.5, yields

$$
\left\|v^{+}\right\|_{L^{2}\left((0, T) \times \mathbb{R}^{N}\right)}^{2}+\left\|v^{-}\right\|_{L^{2}\left((0, T) \times \mathbb{R}^{N}\right)}^{2}=\lim _{\varepsilon \rightarrow 0} \frac{1}{\varepsilon^{N / 2}} \int_{0}^{T} \int_{\mathbb{R}^{N}} \rho\left(x, \frac{x}{\varepsilon}\right)\left|\frac{\partial \Psi_{\varepsilon}}{\partial t}\right|^{2} d x d t .
$$

Finally, recalling $u_{\varepsilon}=\frac{\partial \Psi_{\varepsilon}}{\partial t}+\alpha \Psi_{\varepsilon}$ and using Lemma 6.4 which says that $\Psi_{\varepsilon}$ converges to 0 in a suitable weighted norm, we obtain

$$
\left\|v^{+}\right\|_{L^{2}\left((0, T) \times \mathbb{R}^{N}\right)}^{2}+\left\|v^{-}\right\|_{L^{2}\left((0, T) \times \mathbb{R}^{N}\right)}^{2}=\lim _{\varepsilon \rightarrow 0} \frac{1}{\varepsilon^{N / 2}} \int_{0}^{T} \int_{\mathbb{R}^{N}} \rho\left(x, \frac{x}{\varepsilon}\right)\left|u_{\varepsilon}(t, x)\right|^{2} d x d t,
$$


which is the desired result.

Proof of Lemma 6.2. We begin with the easy case when $\xi^{n} \neq 0$. From the definition (61) of $w_{\varepsilon}$ we have $\chi_{\varepsilon}(\sqrt{\varepsilon} z)=\varepsilon w_{\varepsilon}(z) e^{-2 i \pi \frac{\xi^{n} \cdot z}{\sqrt{\varepsilon}}}$ which, upon differentiating, yields

$$
\left(\nabla_{x} \chi_{\varepsilon}\right)(\sqrt{\varepsilon} z)=\left(\sqrt{\varepsilon} \nabla_{z} w_{\varepsilon}+2 i \pi \xi^{n} w_{\varepsilon}\right) e^{-2 i \pi \frac{\xi^{n} \cdot z}{\sqrt{\varepsilon}}} .
$$

From Lemma 4.1 we know that

$$
\left\|\left(\nabla_{x} \chi_{\varepsilon}\right)(\sqrt{\varepsilon} z)\right\|_{L^{2}\left(\mathbb{R}^{N}\right)} \leq C\left(\left\|v^{0}\right\|_{L^{2}\left(\mathbb{R}^{N}\right)}+\left\|v^{1}\right\|_{H^{2}\left(\mathbb{R}^{N}\right)}\right) .
$$

For $\xi^{n} \neq 0$ there exists a positive constant $C\left(\xi^{n}\right)>0$ (see [12]) such that

$$
\left\|\sqrt{\varepsilon} \nabla_{z} w_{\varepsilon}+2 i \pi \xi^{n} w_{\varepsilon}\right\|_{L^{2}\left(\mathbb{R}^{N}\right)} \geq C\left(\xi^{n}\right)\left(\left\|\sqrt{\varepsilon} \nabla_{z} w_{\varepsilon}\right\|_{L^{2}\left(\mathbb{R}^{N}\right)}+\left\|w_{\varepsilon}\right\|_{L^{2}\left(\mathbb{R}^{N}\right)}\right)
$$

which implies the desired estimate (62). We now study the two-scale convergence of the sequence $w_{\varepsilon}$, which is a solution of

$$
-\left(\sqrt{\varepsilon} \nabla+2 i \pi \xi^{n}\right) \cdot\left(A^{\varepsilon}\left(\sqrt{\varepsilon} \nabla+2 i \pi \xi^{n}\right) w_{\varepsilon}\right)+\varepsilon^{2} \rho^{\varepsilon} w_{\varepsilon}=\rho^{\varepsilon} \psi_{n}^{\varepsilon}\left(\varepsilon v^{0}-v^{1}\right) .
$$

We multiply equation (73) by $\bar{\phi}^{\varepsilon}=\bar{\phi}\left(z, \frac{z}{\sqrt{\varepsilon}}\right)$, where $\phi(z, y)$ is a smooth compactly supported function defined on $\mathbb{R}^{N} \times \mathbb{T}^{N}$. Integrating by parts and using hypothesis $\mathbf{H} \mathbf{2}$ yield

$$
\begin{array}{r}
\int_{\mathbb{R}^{N}}\left[A_{0}^{\varepsilon}+o(1)\right]\left(\sqrt{\varepsilon} \nabla+2 i \pi \xi^{n}\right) w_{\varepsilon} \cdot\left(\sqrt{\varepsilon} \nabla-2 i \pi \xi^{n}\right) \bar{\phi}^{\varepsilon} d z+\varepsilon^{2} \int_{\mathbb{R}^{N}}\left[\rho_{0}^{\varepsilon}+o(1)\right] w_{\varepsilon} \bar{\phi}^{\varepsilon} d z= \\
\int_{\mathbb{R}^{N}}\left[\rho_{0}^{\varepsilon}+o(1)\right] \psi_{n}^{\varepsilon}\left(\varepsilon v^{0}-v^{1}\right) \bar{\phi}^{\varepsilon} d z
\end{array}
$$

Because of the a priori estimate (62), there exist a subsequence and a limit $w_{0}(z, y) \in$ $L^{2}\left(\mathbb{R}^{N} ; H^{1}\left(\mathbb{T}^{N}\right)\right)$ such that $w_{\varepsilon}$ and $\sqrt{\varepsilon} \nabla w_{\varepsilon}$ two-scale converge weakly to $w_{0}$ and $\nabla_{y} w_{0}$ respectively. Passing to the two-scale limit we obtain

$$
-\left(\operatorname{div}_{y}+2 i \pi \xi^{n}\right) A_{0}(y)\left(\nabla_{y}+2 i \pi \xi^{n}\right) w_{0}=-\rho_{0}(y) v^{1}(z) \psi_{n}(y) .
$$

By the simplicity of $\lambda_{n}$, we deduce that $w_{0}$ is given by

$$
w_{0}(z, y)=-\frac{\psi_{n}(y)}{\lambda_{n}} v^{1}(z)
$$

and the uniqueness of this limit implies that the entire sequence converges. The strong twoscale convergence is easily obtained by replacing the test function $\phi^{\varepsilon}$ by $w_{\varepsilon}$ in (74), passing to the limit in the right hand side which implies the convergence of the energies in the left hand side and thus the strong two-scale convergence according to part (2) of Proposition 2.4 .

Now, suppose that $\xi^{n}=0$. The above proof does not work anymore because inequality (72) is not valid for $\xi^{n}=0$. It turns out that $w_{\varepsilon}$ is not any longer bounded in $L^{2}\left(\mathbb{R}^{N}\right)$ but 
is precisely of order $\varepsilon^{-1 / 2}$. To prove this result we can not use simple a priori estimates and we instead rely on a comparison with a four-term asymptotic expansion. We construct a two-scale asymptotic expansion for a version of (73) with purely periodic coefficients (the macroscopic variable $\sqrt{\varepsilon} z$ being replaced by 0 )

$$
-\varepsilon \operatorname{div}_{z}\left(A_{0}\left(\frac{z}{\sqrt{\varepsilon}}\right) \nabla_{z} w_{\varepsilon}^{0}\right)+\varepsilon^{2} \rho_{0}\left(\frac{z}{\sqrt{\varepsilon}}\right) w_{\varepsilon}^{0}=\rho_{0}\left(\frac{z}{\sqrt{\varepsilon}}\right) \psi_{n}\left(\frac{z}{\sqrt{\varepsilon}}\right)\left(\varepsilon v^{0}-v^{1}\right),
$$

where $A_{0}$ and $\rho_{0}$ are defined by (12). Recall that, by virtue of assumption $\mathbf{H 2}$, the difference between $A\left(\sqrt{\varepsilon} z, \frac{z}{\sqrt{\varepsilon}}\right)$ and $A_{0}\left(\frac{z}{\sqrt{\varepsilon}}\right)$ is small (same for $\rho$ ); see (60). The ansatz for $w_{\varepsilon}^{0}$ is taken as

$$
\tilde{w}_{\varepsilon}(z)=\frac{w^{*}(z)}{\sqrt{\varepsilon}}+w^{0}\left(z, \frac{z}{\sqrt{\varepsilon}}\right)+\sqrt{\varepsilon} w^{1}\left(z, \frac{z}{\sqrt{\varepsilon}}\right)+\varepsilon w^{2}\left(z, \frac{z}{\sqrt{\varepsilon}}\right) .
$$

Plugging it in (75) we obtain the following cascade of equations

$$
\begin{aligned}
-\operatorname{div}_{y}\left(A_{0}(y) \nabla_{y} w^{0}(z, y)+A_{0}(y) \nabla_{z} w^{*}(z)\right)= & -\rho_{0}(y) \psi_{n}(y) v^{1}(z) \\
-\operatorname{div}_{y}\left(A_{0}(y) \nabla_{y} w^{1}(z, y)\right)= & \operatorname{div}_{z}\left(A_{0}(y) \nabla_{y} w^{0}(z, y)\right)+\operatorname{div}_{y}\left(A_{0}(y) \nabla_{z} w^{0}\right) \\
& +\operatorname{div}_{z}\left(A_{0}(y) \nabla_{z} w^{*}(z)\right) \\
-\operatorname{div}_{y}\left(A_{0}(y) \nabla_{y} w^{2}(z, y)\right)= & \operatorname{div}_{z}\left(A_{0}(y) \nabla_{y} w^{1}(z, y)\right)+\operatorname{div}_{y}\left(A_{0}(y) \nabla_{z} w^{1}(z, y)\right) \\
& +\operatorname{div}_{z}\left(A_{0}(y) \nabla_{z} w^{0}(z, y)\right)+\rho_{0}(y) \psi_{n}(y) v^{0}(z)
\end{aligned}
$$

The first equation allows us to compute $w^{0}(z, y)$, up to an unknown function $\hat{w}_{0}(z)$,

$$
w^{0}(z, y)=\hat{w}_{0}(z)-\frac{\psi_{n}(y) v^{1}(z)}{\lambda_{n}}+\sum_{i=1}^{N} \frac{\partial w^{*}}{\partial z_{i}}(z) \chi_{i}(y)
$$

where $\chi_{i}, i=1, \ldots, N$, are the usual periodic solutions of the cell problems

$$
-\operatorname{div}_{y}\left(A(y)\left(\nabla_{y} \chi_{i}+e_{i}\right)\right)=0 \quad \text { in } \mathbb{T}^{N}
$$

In order to solve the second equation, we must first check the following compatibility condition

$$
\operatorname{div}_{z}\left(A^{H} \nabla_{z} w^{*}(z)\right)-\frac{1}{\lambda_{n}} \operatorname{div}_{z}\left(v^{1}(z) \int_{\mathbb{T}^{N}} A(y) \nabla_{y} \psi_{n}(y) d y\right)=0,
$$

where $A^{H}$ is the classical homogenized matrix defined by $A^{H} e_{i}=\int_{\mathbb{T}^{N}} A(y)\left(\nabla_{y} \chi_{i}+e_{i}\right) d y$. Equation (76) has a unique (up to an additive constant) solution $w^{*}(z)$ in the space $D^{1,2}\left(\mathbb{R}^{N}\right)=$ $\left\{\phi \in H_{\text {loc }}^{1}\left(\mathbb{R}^{N}\right)\right.$ s.t. $\left.\nabla \phi \in L^{2}\left(\mathbb{R}^{N}\right)^{N}\right\}$. It is not obvious however that $w^{*}$ belongs to $L^{2}\left(\mathbb{R}^{N}\right)$. Since equation (76) has constant coefficients, by Fourier analysis we deduce that

$$
\left|\mathcal{F} w^{*}(\xi)\right| \leq C \frac{\left|\mathcal{F} v^{1}(\xi)\right|}{|\xi|}
$$

where $\mathcal{F} \phi(\xi)$ denotes the Fourier transform of $\phi(x)$. Assumption (28) (see Remark 3.4 for comments) is precisely designed so we can deduce from (77) that indeed $w^{*}$ belongs to 
$L^{2}\left(\mathbb{R}^{N}\right)$. Knowing $w^{*}$ we can compute the solution of the second equation (up to the addition of an unknown function $\left.\hat{w}_{1}(z)\right)$ as

$$
w^{1}(z, y)=\sum_{i=1}^{N} \frac{\partial \hat{w}_{0}}{\partial z_{i}}(z) \chi_{i}(y)+\tilde{w}_{1}(z, y)
$$

where $\tilde{w}_{1}(z, y)$ depends on $z$ only linearly through $\nabla_{z} v^{1}$ and $\nabla_{z} \nabla_{z} w^{*}$ (which himself depends only on $\nabla_{z} v^{1}$ ), but not on $\hat{w}_{0}$.

In order to solve the third equation (for $w^{2}$ ), we must first check the following compatibility condition

$$
\operatorname{div}_{z}\left(A^{H} \nabla_{z} \hat{w}_{0}\right)+\operatorname{div}_{z} \int_{\mathbb{T}^{N}} A(y)\left[\nabla_{y} \tilde{w}_{1}-\frac{\psi_{n}}{\lambda_{n}} \nabla_{z} v^{1}+\sum_{i=1}^{N} \chi_{i} \nabla_{z} \frac{\partial w^{*}}{\partial z_{i}}\right] d y=0
$$

which again admits a unique (up to an additive constant) solution $\hat{w}_{0} \in D^{1,2}\left(\mathbb{R}^{N}\right)$ if $v^{1} \in$ $H^{1}\left(\mathbb{R}^{N}\right)$. Since the coefficients are constant in the above equation, by Fourier analysis we easily check that the $L^{2}\left(\mathbb{R}^{N}\right)$-norm of $\hat{w}_{0}$ is bounded by the $L^{2}\left(\mathbb{R}^{N}\right)$-norm of $v^{1}$.

Since we proved that both $w^{*}$ and $\hat{w}_{0}$ belong to $H^{1}\left(\mathbb{R}^{N}\right)$, it is now obvious that the two-scale asymptotic expansion $\tilde{w}_{\varepsilon}$ satisfies the desired a priori estimate (63), whatever the choices of the underdetermined additive functions $\hat{w}_{1}(z)$ and $\left.\hat{w}_{2}(z)\right)$. To obtain the same for $w_{\varepsilon}$ we bound their difference $\delta_{\varepsilon}(z)=w_{\varepsilon}(z)-\tilde{w}_{\varepsilon}(z)$ which satisfies

$$
-\varepsilon \operatorname{div}_{z}\left(A^{\varepsilon} \nabla_{z} \delta_{\varepsilon}\right)+\varepsilon^{2} \rho^{\varepsilon} \delta_{\varepsilon}=\varepsilon^{3 / 2} f^{\varepsilon}
$$

where

$$
\begin{aligned}
f^{\varepsilon}(z)= & -\rho\left(z, \frac{z}{\sqrt{\varepsilon}}\right)\left(w^{*}(z)+\sqrt{\varepsilon} w^{0}\left(z, \frac{z}{\sqrt{\varepsilon}}\right)+\varepsilon w^{1}\left(z, \frac{z}{\sqrt{\varepsilon}}\right)+\varepsilon^{3 / 2} w^{2}\left(z, \frac{z}{\sqrt{\varepsilon}}\right)\right) \\
& -\varepsilon^{-1 / 2} \operatorname{div}_{z}\left(\left[A_{0}\left(\frac{z}{\sqrt{\varepsilon}}\right)-A\left(\sqrt{\varepsilon} z, \frac{z}{\sqrt{\varepsilon}}\right)\right] \nabla_{z} \tilde{w}_{\varepsilon}\right) \\
& {\left[-\operatorname{div}_{z}\left(A_{0}(y) \nabla_{z} w^{1}(z, y)\right)-\sqrt{\varepsilon} \operatorname{div}_{z}\left(A_{0}(y) \nabla_{z} w^{2}(z, y)\right)\right]\left(y=\frac{z}{\sqrt{\varepsilon}}\right) } \\
& {\left[-\operatorname{div}_{z}\left(A_{0}(y) \nabla_{y} w^{2}(z, y)\right)-\operatorname{div}_{y}\left(A_{0}(y) \nabla_{z} w^{2}(z, y)\right)\right]\left(y=\frac{z}{\sqrt{\varepsilon}}\right) . }
\end{aligned}
$$

We check that

$$
\int_{\mathbb{R}^{N}} f^{\varepsilon} \delta_{\varepsilon} d z \leq C\left(\left\|\delta_{\varepsilon}\right\|_{L^{2}\left(\mathbb{R}^{N}\right)}+\varepsilon^{-1 / 2}\left\|\nabla \delta_{\varepsilon}\right\|_{L^{2}\left(\mathbb{R}^{N}\right)}\right)
$$

because of assumption $\mathbf{H 2}$ and (60). Therefore, we obtain the following inequality

$$
\varepsilon\left\|\nabla_{z} \delta_{\varepsilon}\right\|_{L^{2}\left(\mathbb{R}^{N}\right)^{N}}^{2}+\varepsilon^{2}\left\|\delta_{\varepsilon}\right\|_{L^{2}\left(\mathbb{R}^{N}\right)}^{2} \leq C \sqrt{\varepsilon}\left(\varepsilon\left\|\delta_{\varepsilon}\right\|_{L^{2}\left(\mathbb{R}^{N}\right)}+\sqrt{\varepsilon}\left\|\nabla \delta_{\varepsilon}\right\|_{L^{2}\left(\mathbb{R}^{N}\right)}\right)
$$

which implies

$$
\sqrt{\varepsilon}\left\|\delta_{\varepsilon}\right\|_{L^{2}\left(\mathbb{R}^{N}\right)}+\left\|\nabla \delta_{\varepsilon}\right\|_{L^{2}\left(\mathbb{R}^{N}\right)} \leq C
$$


and, in turn, the desired estimate (63). We pass to the weak two-scale limit in equation (73) with $\xi^{n}=0$ as before. The strong two-scale convergence of $\sqrt{\varepsilon} \nabla w_{\varepsilon}$ is obtained by remarking that $\sqrt{\varepsilon}\left\|\nabla \delta_{\varepsilon}\right\|_{L^{2}\left(\mathbb{R}^{N}\right)} \leq C \sqrt{\varepsilon}$. This completes the proof of the lemma.

Acknowledgments. This work has partially been supported by the ECOS project C04E07 of cooperation between Chile and France, and by the Chair "Mathematical modelling and numerical simulation, F-EADS Ecole Polytechnique INRIA". The first author is partly supported by the DEFI project at INRIA Saclay Ile de France. The second author is partially supported by Grant "Dirección de Investigación", U. del Bío-Bío 073609 1/R and by Grant FONDECYT 1090510.

\section{References}

[1] Allaire G. [1992] Homogenization and two-scale convergence, SIAM J. Math. Anal. 23(6), $1482-1518$.

[2] Allaire G. [2007] Numerical Analysis and Optimization. An Introduction to Mathematical Modelling and Numerical Simulation, Oxford University Press.

[3] Allaire G., Palombaro M. [2006] Localization for the Schrödinger equation in a locally periodic medium, SIAM J. Math Anal. 38, 127-142.

[4] Allaire G., Palombaro M., Rauch J. [2009] Diffractive behavior of the wave equation in periodic media: weak convergence analysis, to appear in Annali di Matematica Pura ed Applicata.

[5] Allaire G., Palombaro M., Rauch J. [2008] Diffractive Geometric Optics for Bloch Wave Packets, submitted.

[6] Allaire G., Piatnitsky A. [2002] Uniform spectral asymptotics for singularly perturbed locally periodic operators Comm. in Partial Differential Equations 27, 705-725.

[7] Anderson P. W., Absence of diffusion in certain random lattices, Phys. Rev. 109, 14921505 (1958).

[8] Benisty H., Weisbuch C., [2006] Photonic crystals, in Progress in Optics 49, pp.177-313, E. Wolf ed., Elsevier.

[9] Bensoussan A., Lions J.L., Papanicolaou G. [1978] Asymptotic Analysis in Periodic Structures, North-Holland, Amsterdam.

[10] Brézis H. [1973] Operateurs maximaux monotones et semi-groupes de contractions dans les espaces de Hilbert, North-Holland, Amsterdam.

[11] Carmona R., Lacroix J. [1990] Spectral Theory of Random Schrödinger Operators, Birkhäuser, Boston. 
[12] Conca C., Planchard J., Vanninathan M., [1995] Fluids and periodic structures, RMA 38, J. Wiley \& Masson, Paris.

[13] Donnat P., Joly J.-L., Metivier G., Rauch J., [1996] Diffractive nonlinear geometric optics, Séminaire sur les Equations aux Dérivées Partielles, 1995-1996, Exp. No. XVII, 25 pp., Ecole Polytechnique, Palaiseau.

[14] Figotin A., Klein A., [1996] Localization of classical waves. I. Acoustic waves, Comm. Math. Phys. 180, no. 2, pp.439-482.

[15] Francfort G., Murat F., [1992] Oscillations and energy densities in the wave equation, Comm. Partial Differential Equations 17, pp.1785-1865.

[16] Gérard P. [1991] Mesures semi-classiques et ondes de Bloch, Séminaire sur les equations aux Dérivées Partielles, 1990-1991, Exp. No. XVI, 19 pp., École Polytech., Palaiseau.

[17] Gérard P., Markowich P., Mauser N., Poupaud F. [1997] Homogenization limits and Wigner transforms, Comm. Pure Appl. Math. 50, no. 4, pp.323-379.

[18] Kato T. [1980] Perturbation Theory for Linear Operators, Springer-Verlag, Berlin, Heidelberg.

[19] Martin de Sterke C., Sipe J., [1998] Envelope-function approach for the electrodynamics of nonlinear periodic structures, Phys. Rev. A, 38, pp.5149-5165.

[20] Nguetseng G. [1989] A general convergence result for a functional related to the theory of homogenization, SIAM J. Math. Anal. 20 (3), 608-623.

[21] Pazy A. [1983] Semigroups of linear operators and applications to partial differential equations, Applied Mathematical Sciences, 44, Springer-Verlag, New York.

[22] Reed M., Simon B. [1972-78] Methods of Modern Mathematical Physics. I. Functional Analysis, II. Fourier Analysis and Self-Adjointness, III. Scattering Theory, IV. Analysis of Operators, Academic Press, New York.

[23] Russell P. St. J., [2006] Photonic crystal fibers, J. Lightwave. Technol., 24 (12), pp.47294749 .

[24] Sipe J., Winful H., [1988] Nonlinear Schrödinger solitons in a periodic structure, Optics Letters, 13, pp.132-133. 